\title{
CENTERS OF GENERIC HECKE ALGEBRAS
}

\author{
LENNY K. JONES
}

ABstract. Let $W$ be a Weyl group and let $W^{\prime}$ be a parabolic subgroup of $W$. Define $A$ as follows:

$$
A=R \otimes_{\mathrm{Q}[u]} \mathscr{A}(W)
$$

where $\mathscr{A}(W)$ is the generic algebra of type $A_{n}$ over $\mathrm{Q}[u], u$ an indeterminate, associated with the group $W$, and $R$ is a $\mathbf{Q}[u]$-algebra, possibly of infinite rank, in which $u$ is invertible. Similarly, we define $A^{\prime}$ associated with $W^{\prime}$. Let $M$ be an $A-A$ bimodule, and let $b \in M$. Define the relative norm [14]

$$
N_{W, W^{\prime}}(b)=\sum_{t \in T} u^{-l(t)} a_{t^{-1}} b a_{t}
$$

where $T$ is the set of distinguished right coset representives for $W^{\prime}$ in $W$. We show that if $b \in Z_{M}\left(A^{\prime}\right)=\left\{m \in M \mid m a^{\prime}=a^{\prime} m \forall a^{\prime} \in A^{\prime}\right\}$, then $N_{W, W^{\prime}}(b) \in Z_{M}(A)$. In addition, other properties of the relative norm are given and used to develop a theory of induced modules for generic Hecke algebras including a Markey decomposition. This section of the paper is previously unpublished work of P. Hoefsmit and L. L. Scott.

Let $\alpha=\left(k_{1}, k_{2}, \ldots, k_{z}\right)$ be a partition of $n$ and let $S_{\alpha}=\prod_{i=1}^{Z} S_{k_{i}}$ be a "left-justified" parabolic subgroup of $S_{n}$ of shape $\alpha$.

Define

$$
b_{\alpha}=N_{S_{n}, S_{\alpha}}\left(\mathscr{N}_{\alpha}\right) \text {, }
$$

where

$$
\mathcal{N}_{\alpha}=\prod_{i=1}^{Z} N_{S_{k_{i}-1}, S_{1}}\left(a_{w_{i}}\right)
$$

with $w_{i}$ a $k_{i}$-cycle of length $k_{i}-1$ in $S_{k_{i}}$. Then the main result of this paper is

Theorem. The set $\left\{b_{\alpha} \mid \alpha \vdash n\right\}$ is a basis for $Z_{A\left(S_{n}\right)}\left(A\left(S_{n}\right)\right)$ over $\mathbf{Q}\left[u, u^{-1}\right]$.

Remark. The norms $b_{\alpha}$ in $Z_{A\left(S_{n}\right)}\left(A\left(S_{n}\right)\right)$ are analogs of conjugacy class sums in the center of $\mathbf{Q} S_{n}$ and, in fact, specialization of these norms at $u=1$ gives the standard conjugacy class sum basis of the center of $\mathbf{Q} S_{n}$ up to coefficients from $\mathbf{Q}$.

\section{Preliminaries}

This section contains certain key definitions and results, presented without proofs, of standard fare needed for later sections.

Received by the editors June 24, 1988. Presented to the Society, January 1988 in Atlanta, Georgia.

1980 Mathematics Subject Classification (1985 Revision). Primary 16A45, 16A64; Secondary 20C05, 20C 30 .

Key words and phrases. Generic Hecke algebras, Weyl groups, parabolic subgroups. 
1.1. Parabolic subgroups of Weyl groups. Let $W=W(\Delta)$ be a Weyl group and let $R=\left\{w_{\alpha_{1}}, w_{\alpha_{2}}, \ldots, w_{\alpha_{n}}\right\}$ with $\alpha_{1}, \alpha_{2}, \ldots, \alpha_{n}$ fundamental.

Definition (1.1.1). Let $J \leq R$. Then $W_{j}=\langle J\rangle$ is a parabolic subgroup of $W$.

Proposition (1.1.2). Let $I, J \leq R$. Let $W_{I} w W_{J}$ be a double coset in $W$. Then there is a unique element $\tilde{w}$ of minimal length in $W_{I} w W_{J}$ such that, given any $v \in W_{I} w W_{J}$, there exist elements $s \in W_{I}$ and $t \in W_{J}$ so that $v=s \tilde{w} t$, with $l(s \tilde{w} t)=l(s)+l(\tilde{w})+l(t)$.

Definition (1.1.3). The element $\tilde{w}$ in Proposition (1.1.2) is called a distinguished double coset representative.

Note. If $J=\varnothing$, then $\tilde{w}$ is called a distinguished right coset representative, adopting the convention $W_{\varnothing}=\{1\}$.

Proposition (1.1.4). Let $I, J<R$ and let $\tilde{w}$ be distinguished in $W_{I} w W_{J}$. Then $\left(W_{I}\right)^{\tilde{w}} \cap W_{J}$ is a parabolic subgroup of $W$.

Theorem (1.1.5) (Exchange condition). If $w_{\alpha_{1}} w_{\alpha_{2}} \cdots w_{\alpha_{k}}$ is reduced and

$$
w_{\alpha_{0}} w_{\alpha_{1}} w_{\alpha_{2}} \cdots w_{\alpha_{k}}
$$

is not reduced $\left(\alpha_{0} \in \Pi\right)$, then for some $i \leq k$,

$$
w_{\alpha_{1}} w_{\alpha_{2}} \cdots w_{\alpha_{i}}=w_{\alpha_{0}} w_{\alpha_{1}} w_{\alpha_{2}} \cdots w_{\alpha_{i-1}} .
$$

Remark. Given $w_{\alpha_{1}} w_{\alpha_{2}} \cdots w_{\alpha_{k}}$ reduced with $w_{\alpha_{0}} w_{\alpha_{1}} w_{\alpha_{2}} \cdots w_{\alpha_{k}}$ not reduced, the exchange condition implies that

$$
\begin{aligned}
\left(w_{\alpha_{0}} w_{\alpha_{1}} \cdots w_{\alpha_{i-1}}\right) w_{\alpha_{i}} w_{\alpha_{i+1}} \cdots w_{\alpha_{k}} & =\left(w_{\alpha_{1}} \cdots w_{\alpha_{i}}\right) w_{\alpha_{i}} w_{\alpha_{i+1}} \cdots w_{\alpha_{k}} \\
& =w_{\alpha_{1}} \cdots w_{\alpha_{i-1}} w_{\alpha_{i+1}} \cdots w_{\alpha_{k}} .
\end{aligned}
$$

1.2. The generic algebra and specialized algebra. Let $(W, R)$ be a finite Coxeter system with $R=\left\{w_{1}, w_{2}, \ldots, w_{n}\right\}$. Let $D=\mathbf{Q}\left[u_{1}, u_{2}, \ldots, u_{n}\right]$ be the polynomial ring over $\mathbf{Q}$ in indeterminates $\left\{u_{i}\right\}$ corresponding to the distinguished generators in $R$ and satisfying the condition that $u_{i}=u_{j}$ whenever $w_{i}$ and $w_{j}$ are conjugate in $W$.

Definition (1.2.1). The generic algebra (or generic Hecke algebra) $\mathscr{A}=\mathscr{A}(W)$ is the algebra over $D$, with basis $\left\{a_{w} \mid w \in W\right\}$ over $D$, identity element $a_{1}$, and multiplication defined by the conditions

$$
a_{w_{i}} a_{w}= \begin{cases}a_{w_{i} w}, & l\left(w_{i} w\right) \geq l(w), \\ u_{i} a_{w_{i} w}+\left(u_{i}-1\right) a_{w}, & l\left(w_{i} w\right)<l(w) .\end{cases}
$$

$\mathscr{A}$ has a presentation with generators $\left\{a_{w_{i}} \mid w_{i} \in R\right\}$ and quadratic relations

$$
a_{w_{i}}^{2}=u_{i} a_{1}+\left(u_{i}-1\right) a_{w_{i}}, \quad w \in R,
$$

and homogeneous relations

$$
a_{w_{i}} a_{w_{j}} a_{w_{i}} \cdots=a_{w_{j}} a_{w_{i}} a_{w_{j}} \ldots \quad(i \neq j)
$$


with $n_{i j}$ factors, where $n_{i j}$ is the order of the product $w_{i} w_{j}$ in $W$. Thus we may define the following homomorphism:

$$
\text { ind } \begin{aligned}
: \mathscr{A} & \rightarrow D \\
a_{w_{i}} & \mapsto u_{i} .
\end{aligned}
$$

We then may define the Poincaré polynomial of $W$, and generic degree corresponding to an irreducible character $\chi$ of $\bar{K} \otimes_{R} \mathscr{A}(W)$ where $\bar{K}$ is an algebraic closure of $K$.

Definition (1.2.2). Let $\mathscr{A}=\mathscr{A}(W)$ be a generic algebra, and $\chi$ an irreducible character of $\bar{K} \otimes_{R} \mathscr{A}$. The generic degree corresponding to $\chi$ is the element of $\bar{K}$ given by

$$
d_{\chi}=\frac{(\operatorname{deg} \chi) \cdot d_{W}}{\sum_{w \in W}\left(\operatorname{ind}\left(a_{w}\right)\right)^{-1} \chi\left(a_{w}\right) \chi\left(a_{w^{-1}}\right)},
$$

where $d_{W}$ is the Poincare polynomial of $W$ and is defined by

$$
d_{W}=\sum_{w \in W} \operatorname{ind}\left(a_{w}\right) .
$$

Definition (1.2.3). Let $L$ be any field of characteristic zero and $\phi: D \rightarrow L$ a homomorphism. Consider $L$ as an $L-D$ bimodule by setting

$$
l \lambda d=l \lambda \phi(d), \quad l, \lambda \in L, d \in D .
$$

Then $\mathscr{A}_{f, L}=L \otimes_{D} \mathscr{A}$ is an associative algebra over $L$ called a specialized algebra.

$\mathscr{A}_{f, L}$ has a basis $\left\{a_{w \phi}=1 \otimes a_{w} \mid w \in W\right\}$, generators $\left\{a_{w_{i} \phi} \mid w_{i} \in R\right\}$, and defining relations obtained by applying $\phi$ to the relations for $\mathscr{A}$ in Definition (1.2.1).

Proposition (1.2.4). Let $f: D \rightarrow \mathbf{Q}$ be defined by $f\left(u_{i}\right)=q_{i} \forall i$. Then $\mathscr{A}_{f, \mathbf{Q}} \simeq$ $\mathscr{H}(G, B)$.

Let $f: D \rightarrow \mathbf{Q}$ be defined by $f\left(u_{i}\right)=1 \forall i$. Then $\mathscr{A}_{f, \mathbf{Q}} \simeq \mathbf{Q} W$.

Theorem (1.2.5) (Tits). Let $G=\operatorname{SL}(n, q), B=$ subgroup of $G$ consisting of upper triangular matrices and $W=S_{n}$. Then $\mathscr{H}(G, B) \simeq \mathbf{C W}$.

Remark. In [11], Curtis and Scott give a (modular-theoretic) proof of the fact that $\mathscr{H}(G, B) \simeq \mathbf{Q} W$, with $G, B$ and $W$ as above, along the lines of Feit [12] which motivated the work of Hoefsmit and Scott [14] presented in the next section.

\section{General Results}

The material in this section is due mostly to Peter Hoefsmit and Leonard Scott, taken from unpublished work [14] done in 1976. We would like at this time to point out the results of this section which the reader should focus on in order to understand the main results of this paper, given in $\S 3$. They are Lemma (2.12), Proposition (2.13), and Theorem (2.30). 
Definition (2.1). Let $u$ be an indeterminate. Define

$$
A=R \otimes_{\mathrm{Q}[u]} \mathscr{A},
$$

where $R$ is a $\mathbf{Q}[u]$-algebra, possibly of infinite rank, in which $u$ is invertible and where $\mathscr{A}=\mathscr{A}(W)$ is the generic algebra of a Weyl group $W$ over $\mathbf{Q}[u]$. Similarly, define

$$
A^{\prime}=R \otimes_{\mathbf{Q}[u]} \mathscr{A}^{\prime},
$$

where $\mathscr{A}^{\prime}=\mathscr{A}^{\prime}\left(W^{\prime}\right)$ is the generic subalgebra of $\mathscr{A}$ associated with a parabolic subgroup $W^{\prime}$ of $W$.

Definition (2.2). Let $M$ be an $A^{\prime}-A^{\prime}$ bimodule. Define

$$
Z_{M}\left(A^{\prime}\right)=\left\{m \in M \mid a^{\prime} m=m a^{\prime} \text { for all } a^{\prime} \in A^{\prime}\right\} .
$$

Note. We will write $Z(A)$ for $Z_{A}(A)$.

Remark. If $W_{1}$ and $W_{2}$ are parabolic subgroups of $W$, then $A_{1}$ and $A_{2}$ will denote the corresponding subalgebras of $A$ unless otherwise stated. Also, given an arbitrary parabolic subgroup $P$ of $W$, we will sometimes denote the corresponding subalgebra of $A$ as $A(P)$.

Definition (2.3). Let $M$ be an $A-A$ bimodule and let $b \in M$. Let $R$ be the set of distinguished right coset representatives for $W^{\prime}$ in $W$. Define the relative norm

$$
N_{W, W^{\prime}}(b)=\sum_{r \in R} u^{-l(r)} a_{r^{-1}} b a_{r} .
$$

Note. If $W^{\prime}$ is the identity subgroup, we will write $N_{W, 1}(b)$.

Lemma (2.4). Let $M$ be an $A$-A bimodule, and let $b \in M$. Then $N_{W, 1}(b) \in$ $Z_{M}(A)$.

Proof. Consider the $A-A$ bimodule $M \oplus A$. For each $m \in M$ we can define $\phi_{m} \in \operatorname{Hom}_{\mathbf{Q}(u)}(M \oplus A, M \oplus A)$ by

$$
\phi_{m}\left(m^{\prime}+a\right)=m a \text { for any } m^{\prime} \in M .
$$

Thus $M \leq \operatorname{Hom}_{\mathbf{Q}(u)}(M \oplus A, M \oplus A)$, and since $N_{W, 1}(b) \in M$, we have $N_{W, 1}(b) \in \operatorname{Hom}_{\mathbf{Q}(u)}(M \oplus A, M \oplus A)$. Now $\left\{a_{w} \mid w \in W\right\}$ and $\left\{u^{-l(w)} a_{w^{-1}} \mid w \in\right.$ $W\}$ are dual bases for the symmetric algebra $\mathbf{Q}(u) A$ with respect to the nondegenerate associative coefficient at the identity form. Hence $\mathbf{Q}(u) A$ is Frobenius and therefore $N_{W, 1}(b) \in \operatorname{Hom}_{\mathbf{Q}(u) A}(M \oplus A, M \oplus A)$ [8]. In particular, $a N_{W, 1}(b)=N_{W, 1}(b) a$ for all $a \in A$. Hence, $N_{W, 1}(b) \in Z_{M}(A)$.

Lemma (2.5). Let $a_{1}$ be the identity in $A$. Then $N_{W, 1}\left(a_{1}\right)$ is invertible in $\mathbf{Q}(u) A$. (Note. By [13], $\mathbf{Q}(u)$ is a splitting field for $A$ when $\mathscr{A}(w)$ is type $A_{n}$; for other situations extend to a splitting field.)

Proof. Write

$$
N_{W, 1}\left(a_{1}\right)=\sum_{w \in W} u^{-l(t)} a_{w^{-1}} a_{w}=\sum_{\chi} c_{\chi} e_{\chi},
$$


where the $e_{\chi}$ are the central primitive idempotents associated with the irreducible characters for $A$ over $\mathbf{Q}(u)$. We will determine $c_{\chi}$ explicitly. Applying a particular character $\chi$ to the norm, we get

$$
\sum_{w \in W} u^{-l(w)} \chi\left(a_{w^{-1}} a_{w}\right)=c_{\chi} \chi\left(a_{1}\right)
$$

Let $\left\{X_{i j} \mid 1 \leq i, j \leq \chi\left(a_{1}\right)\right\}$ be a set of matrix coordinate functions associated with $\chi$ and let $\left\{f_{i j} \mid 1 \leq i, j \leq \chi\left(a_{1}\right)\right\}$ be the set of corresponding matrix units. Then

$$
\begin{aligned}
\sum_{w \in W} u^{-l(w)} \chi\left(a_{w^{-1}} a_{w}\right) & =\sum_{w \in W} \sum_{i, j} u^{-l(w)} X_{j i}\left(a_{w^{-1}}\right) X_{i j}\left(a_{w}\right) \\
& =\sum_{w \in W} \sum_{i, j} u^{-l(w)} \chi\left(f_{i j} a_{w^{-1}}\right) X_{i j}\left(a_{w}\right) .
\end{aligned}
$$

Now write $f_{i j}=\sum_{w \in W} c_{w} a_{w}$. Consider the function $\rho=\sum_{\chi} d_{\chi} \chi$, where $d_{\chi}$ is the generic degree of $\chi$. Observe that

$$
\rho\left(a_{w}\right)= \begin{cases}d_{W} & \left(\text { where } d_{W} \text { is the Poincare polynomial for } W\right) \\ & \text { if } w=\text { the identity } \\ 0 & \text { otherwise. }\end{cases}
$$

Let $\tau \in W$. Then

$$
\rho\left(f_{i j} a_{\tau}\right)=\rho\left(\left(\sum_{w \in W} c_{w} a_{w}\right) a_{\tau}\right)= \begin{cases}c_{w} u^{l(w)} d_{W} & \text { if } \tau=w^{-1} \\ 0 & \text { otherwise }\end{cases}
$$

Also,

$$
\rho\left(f_{i j} a_{w^{-1}}\right)=d_{\chi} \chi\left(f_{i j} a_{w^{-1}}\right) .
$$

Thus

$$
c_{w}=d_{W}^{-1} d_{\chi} u^{-l(w)} \chi\left(f_{i j} a_{w^{-1}}\right)
$$

and hence

$$
f_{i j}=\sum_{w \in W} d_{W}^{-1} d_{\chi} u^{-l(w)} \chi\left(f_{i j} a_{w^{-1}}\right) a_{w} .
$$

Multiplying both sides of $(2.8)$ by $f_{j i}$ and applying $\chi$ gives

$$
\chi\left(f_{j i} f_{i j}\right)=\sum_{w \in W} d_{W}^{-1} d_{\chi} u^{-l(w)} \chi\left(f_{i j} a_{w^{-1}}\right) \chi\left(f_{j i} a_{w}\right) .
$$

Summing both sides of (2.9) over $i$ and $j$, we have

$$
\chi\left(a_{1}\right)^{2}=\sum_{i, j} \sum_{w \in W} d_{W}^{-1} d_{\chi} u^{-l(w)} \chi\left(f_{i j} a_{w^{-1}}\right) \chi\left(f_{j i} a_{w}\right),
$$

since $f_{j i} f_{i j}=f_{j j}$. Combining (2.10) with (2.7) yields

$$
\chi\left(a_{1}\right)^{2}=d_{W}^{-1} d_{\chi} \sum_{w \in W} u^{-l(w)} \chi\left(a_{w^{-1}} a_{w}\right)
$$


Finally, from (2.11) and (2.6) we deduce that $c_{\chi}=d_{W}^{-1} d_{\chi} \chi\left(a_{1}\right)$, which is invertible in $\mathbf{Q}(u)$ and the lemma follows.

We see that our norm has a nice transitivity property with respect to parabolic subgroups.

Lemma (2.12). Let $M$ be an $A-A$ bimodule and let $b \in M$. Let $W^{\prime}$ and $W^{\prime \prime}$ be parabolic subgroups of $W$ such that $W^{\prime} \leq W^{\prime \prime} \leq W$. Then

$$
N_{W, W^{\prime}}(b)=N_{W, W^{\prime \prime}}\left(N_{W^{\prime \prime}, W^{\prime}}(b)\right) \text {. }
$$

Proof. Let $R$ be the set of distinguished right coset representatives for $W^{\prime \prime}$ in $W$, let $T$ be the set of distinguished right coset representatives for $W^{\prime}$ in $W^{\prime \prime}$ and let $S$ be the set of distinguished right coset representatives for $W^{\prime}$ in $W$. Write

$$
W=\bigcup_{r \in R} W^{\prime \prime} r=\bigcup_{r \in R}\left(\bigcup_{t \in T} W^{\prime} t\right) r .
$$

From this decomposition we see that $t r \in S$. That is, since $r$ is distinguished in $W^{\prime \prime} r$ and $t$ is distinguished in $W^{\prime} t$, it follows that $t r$ is distinguished in $W^{\prime} t r$. Also, from Proposition (1.1.2), we have $l(t r)=l(t)+l(r)$ since $t \in W^{\prime \prime}$. Thus

$$
\begin{aligned}
N_{W, W^{\prime \prime}}\left(N_{W^{\prime \prime}, W^{\prime}}(b)\right) & =\sum_{r \in R} u^{-l(r)} a_{r^{-1}}\left[\sum_{t \in T} u^{-l(t)} a_{t^{-1}} b a_{t}\right] a_{r} \\
& =\sum_{r \in R} \sum_{t \in T} u^{-l(t r)} a_{r^{-1} t^{-1}} b a_{t r} \\
& =\sum_{s \in S} u^{-l(a)} a_{s^{-1}} b a_{s}=N_{W, W^{\prime}}(b) .
\end{aligned}
$$

Proposition (2.13). Let $M$ be an $A$-A bimodule and let $b \in Z_{M}\left(A^{\prime}\right)$. Then

(1) $N_{W, W^{\prime}}(b) \in Z_{M}(A)$;

(2) If $Z_{M}(A)$ is regarded as a $Z(A)$-module, then $N_{W, W^{\prime}}\left(Z_{M}\left(A^{\prime}\right)\right)$ is a $Z(A)$-submodule of $Z_{M}(A)$.

Proof. (1) Clearly $N_{W, W^{\prime}}(b) \in M$. Now

$$
\begin{aligned}
N_{W, W^{\prime}}(b) & =N_{W, W^{\prime}}\left(\frac{N_{W^{\prime}, 1}\left(a_{1}\right)}{N_{W^{\prime}, 1}\left(a_{1}\right)} b\right) \quad \text { by Lemma }(2.5) \\
& =N_{W, W^{\prime}}\left(N_{W^{\prime}, 1}\left(\frac{a_{1}}{N_{W^{\prime}, 1}\left(a_{1}\right)}\right) b\right) \text { by Lemma }(2.4) \\
& =N_{W, W^{\prime}}\left(N_{W^{\prime}, 1}\left(\frac{b}{N_{W^{\prime}, 1}\left(a_{1}\right)}\right)\right) \text { since } b \in Z_{M}\left(A^{\prime}\right) \\
& =N_{W, 1}\left(\frac{b}{N_{W^{\prime}, 1}\left(a_{1}\right)}\right) \text { by Lemma }(2.12) .
\end{aligned}
$$


But $N_{W, 1}\left(b / N_{W^{\prime}, 1}\left(a_{1}\right)\right) \in Z_{\mathbf{Q}(u) A \cdot M}(A)$ by Lemma (2.4), and since

$$
Z_{\mathbf{Q}(u) A \cdot M}(A) \leq Z_{\mathbf{Q}(u) M}(A),
$$

we have

$$
N_{W, W^{\prime}}(b) \in M \cap Z_{\mathbf{Q}(u) M}(A)=Z_{M}(A),
$$

which proves (1).

(2) For $z \in Z(A)$, we have

$$
N_{W, W^{\prime}}(b) z=N_{W, W^{\prime}}(b z),
$$

which proves $(2)$.

Lemma (2.14). Let $a_{w}$ be any basis element of $A$. Then $a_{w}$ is invertible in $A$. Proof. Since $w$ can be expressed as a product of fundamental reflections, we may assume without loss of generality that $w$ itself is a fundamental reflection. From the relation $a_{w}^{2}=u a_{1}+(u-1) a_{w}$, we easily derive

$$
a_{w}^{-1}=u^{-1}\left(a_{w}-(u-1) a_{1}\right) \in A \text {. }
$$

Lemma (2.15). Let $W_{1}$ and $W_{2}$ be two parabolic subgroups of $W$ such that $W_{2}=W_{1}^{u \prime}$ for some $w \in W$. Let $N$ be an $A-A_{1}$ bimodule. Consider the $A-A_{2}$ bimodule $N \otimes_{A_{1}} a_{d}$ where $d$ is distinguished in $W_{1} w W_{2}=W_{1} w=w W_{2}$. Then

$$
N \otimes_{A_{1}} A \simeq\left(N \otimes_{A_{1}} a_{d}\right) \otimes_{A_{2}} A .
$$

Proof. First note that $W_{2}=W_{1}^{d}$ so that $W_{1} d W_{2}=W_{1} d=W_{2}$ and $N \otimes_{A_{1}} a_{d}$ really is an $A-A_{2}$ bimodule. Now $\left(N \otimes_{A_{1}} a_{d}\right) \otimes_{A_{2}} A \simeq N \otimes_{A_{1}}\left(A_{1} a_{d} \otimes_{A_{2}} A\right)$, so it will be enough to show that $A_{1} a_{d} \otimes_{A_{2}} A \simeq A$. Define maps

$$
\begin{aligned}
\phi: A_{1} a_{d} \otimes_{A_{2}} A & \rightarrow A & \psi: A & \rightarrow A_{1} a_{d} \otimes_{A_{2}} A \\
a^{\prime} a_{d} \otimes b & \mapsto a^{\prime} a_{d} b, & a & \mapsto a_{d} \otimes a_{d}^{-1} a .
\end{aligned}
$$

Since $a_{d}^{-1} \in A$ by Lemma (2.14), $\psi$ makes sense. Let $\hat{a} \in A_{1}$ and $\tilde{a} \in A$. Then $\hat{a} a_{d}=a_{d} \bar{a}$ for some $\bar{a} \in A_{2}$. Thus

$$
\phi\left(\hat{a} \cdot a^{\prime} a_{d} \otimes b\right)=\hat{a} a^{\prime} a_{d} b=\hat{a} \cdot \phi\left(a^{\prime} a_{d} \otimes b\right),
$$

and

$$
\phi\left(a^{\prime} a_{d} \otimes b \cdot \tilde{a}\right)=a^{\prime} a_{d} b \tilde{a}=\phi\left(a^{\prime} a_{d} \otimes b\right) \cdot \tilde{a},
$$

which shows that $\phi$ is an $A_{1}-A$ bimodule map. Also

$$
\begin{aligned}
\psi(\hat{a} \cdot a) & =a_{d} \otimes a_{d}^{-1} \hat{a} a=a_{d} \otimes \bar{a} a_{d}^{-1} a \\
& =a_{d} \bar{a} \otimes a_{d}^{-1} a=\hat{a} a_{d} \otimes a_{d}^{-1} a=\hat{a} \psi(a)
\end{aligned}
$$

and

$$
\psi(a \cdot \tilde{a})=a_{d} \otimes a_{d}^{-1} a \tilde{a}=\psi(a) \cdot \tilde{a}
$$


so that $\psi$ is an $A_{1}-A$ bimodule map. Finally,

$$
(\phi \circ \psi)(a)=\phi\left(a_{d} \otimes a_{d}^{-1} a\right)=a
$$

and

$$
\begin{aligned}
(\psi \circ \phi)\left(\hat{a} a_{d} \otimes b\right) & =\psi\left(\hat{a} a_{d} b\right)=a_{d} \otimes a_{d}^{-1} \hat{a} a_{d} b \\
& =a_{d} a_{d}^{-1} \hat{a} a_{d} \otimes b \quad \text { since } a_{d}^{-1} \hat{a} a_{d} \in A_{2}, \\
& =\hat{a} a_{d} \otimes b,
\end{aligned}
$$

and the lemma follows.

Lemma (2.16). Let $R$ be the set of distinguished right coset representatives for $W^{\prime}$ in $W$. Then

$$
A=\bigoplus_{r \in R} A^{\prime} a_{r} .
$$

Proof. This follows immediately from the decomposition $W=\dot{U}_{r \in R} W_{r}^{\prime}$ and Proposition (1.1.2).

Lemma (2.17). Let $W_{1}$ and $W_{2}$ be parabolic subgroups of $W$ and let $W_{1} d W_{2}$ be a double coset in $W$ with $d$ distinguished. Then for any $w_{1} \in W_{1}$, we have $l\left(w_{1} d\right)=l\left(w_{1}\right)+l(d)$. Similarly, for any $w_{2} \in W_{2}$, we have $l\left(d w_{2}\right)=$ $l(d)+l\left(w_{2}\right)$.

Proof. We give the proof for $W_{2}$. Suppose there is $v \in W_{2}$ such that $l(d v)<$ $l(d)+l(v)$. Write $v=v_{1} v_{2} \cdots v_{z}$ as a reduced product of fundamental reflections $v_{i}$. Then there is a smallest subscript, say $j$, such that $l\left(d v_{1} v_{2} \cdots v_{j-1}\right)=$ $l(d)+l\left(v_{1} \cdots v_{j-1}\right)$, but $l\left(d v_{1} v_{2} \cdots v_{j}\right)=l(d)+l\left(v_{1} \cdots v_{j-1}\right)-1$. Let $\hat{v}=$ $v_{1} v_{2} \cdots v_{j-1}$. By Theorem (1.1.5), we have

$$
d \hat{v} v_{j}= \begin{cases}d \hat{v}^{\prime}, & \text { where } l\left(\hat{v}^{\prime}\right)=l(\hat{v})-1 \\ & \text { if the exchange takes place in } \hat{v} \\ d^{\prime} \hat{v}, & \text { where } l\left(d^{\prime}\right)=l(d)-1 \\ & \text { if the exchange takes place in } d\end{cases}
$$

The first case cannot happen since $l\left(\hat{v} v_{j}\right)=l(\hat{v})+1$. If $d \hat{v} v_{j}=d^{\prime} \hat{v}$ with $l\left(d^{\prime}\right)=l(d)-1$, then $d^{\prime}=d \hat{v} v_{j} \hat{v}^{-1} \in W_{1} d W_{2}$, which contradicts the fact that $d$ is distinguished. This proves the lemma.

Remark. Let $d \in W_{1} d W_{2}$ be distinguished. If $d^{-1} \in W_{2} d^{-1} W_{1}$ is not distinguished, then for some $w_{2} \in W_{2}, w_{1} \in W_{1}$ we have

$$
l(d)=l\left(d^{-1}\right)>l\left(w_{2} d^{-1} w_{1}\right)=l\left(\left(w_{2} d^{-1} w_{1}\right)^{-1}\right)=l\left(w_{1}^{-1} d w_{2}^{-1}\right),
$$

contradicting the fact that $d$ is distinguished. 
Definition (2.18). Let $T$ be a right $A$-module. Then $T$ is induced from a right $A^{\prime}$-module $T^{\prime}$ if $T \simeq T^{\prime} \otimes_{A^{\prime}} A$.

Lemma (2.19). Let $T$ be a right A-module. Let $R$ be the set of distinguished right coset representatives for $W^{\prime}$ in $W$. Then $T$ is induced from the right $A^{\prime}$-module $T^{\prime}$ if and only if $T=\bigoplus_{r \in R} S a_{r}$ for some right $A^{\prime}$-submodule $S$ of $T$ with $S \simeq T^{\prime}$.

Proof. Assume first that $T \simeq T^{\prime} \otimes_{A^{\prime}} A$ and let $\phi$ be an isomorphism from $T^{\prime} \otimes_{A^{\prime}} A$ to $T$. By Lemma (2.16) we have $T^{\prime} \otimes_{A^{\prime}} A=\bigoplus_{r \in R}\left(T^{\prime} \otimes_{A^{\prime}} a_{1}\right) a_{r}$ so take $S$ to be $\phi\left(T^{\prime} \otimes_{A^{\prime}} a_{1}\right)$.

Now assume that $T=\bigoplus_{r \in R} S a_{r}$. We have

$$
\begin{aligned}
\bigoplus_{r \in R} S a_{r} & \simeq \bigoplus_{r \in R}\left(S \otimes_{A^{\prime}} a_{r}\right) \simeq S \otimes_{A^{\prime}}\left(\bigoplus_{r \in R} A a_{r}\right) \\
& =S \otimes_{A^{\prime}} A \text { by Lemma }(2.16)
\end{aligned}
$$

and the lemma follows.

Corollary (2.20). Let $W_{1}$ and $W_{2}$ be parabolic subgroups of $W$ and let $W_{1} d W_{2}$ be a double coset in $W$ with $d$ distinguished. Then the $A_{1}-A_{2}$ bimodule $A_{1} a_{d} A_{2}$ is isomorphic to $A_{1} a_{d} \otimes_{A\left(W_{1}^{d} \cap W_{2}\right)} A_{2}$.

Proof. Let $T$ be the set of distinguished right coset representatives for $W^{\prime}=$ $W_{1}^{d} \cap W_{2}$ in $W_{2}$. Then

$$
\begin{aligned}
A_{1} a_{d} A_{2} & =A_{1} a_{d}\left(\bigoplus_{t \in T} A^{\prime} a_{t}\right) \quad \text { by Lemma (2.16) } \\
& =\bigoplus_{t \in T} A_{1} a_{d} A^{\prime} a_{t} .
\end{aligned}
$$

Let $w^{\prime} \in W^{\prime}$. Then $w^{\prime}=d^{-1} w_{1} d$ for some $w_{1} \in W_{1}$. By Lemma (2.17), $l\left(w_{1} d\right)=l\left(w_{1}\right)+l(d)$. Also, since $w^{\prime} \in W_{2}, l\left(d w^{\prime}\right)=l(d)+l\left(w^{\prime}\right)$ by Lemma (2.17). Thus there is a corresponding relation in $A$. In particular, given an element in $A_{1} a_{d} A^{\prime}$, say $\alpha a_{d} \alpha^{\prime}$, with $\alpha \in A_{1}, \alpha^{\prime} \in A^{\prime}$, we may write it as $\alpha \hat{\alpha} a_{d}$ for some $\hat{\alpha} \in A_{1}$. Therefore $A_{1} a_{d} A^{\prime} \leq A_{1} a_{d}$. Clearly $A_{1} a_{d} \leq A_{1} a_{d} A^{\prime}$ and so $A_{1} a_{d} A^{\prime}=A_{1} a_{d}$. Hence $\bigoplus_{t \in T} A_{1} a_{d} A^{\prime} a_{t}=\bigoplus_{t \in T} A_{1} a_{d} a_{t}$. Now apply Lemma (2.19) with $S=A_{1} a_{d}$ to get the result.

Lemma (2.21). Let $W_{1}$ and $W_{2}$ be parabolic subgroups of $W$. Let $D$ be the set of distinguished $W_{1}-W_{2}$ double coset representatives in $W$. Let $R$ be the set of distinguished right coset representatives for $W_{1}$ in $W$. For $d \in D$, let $T_{d}$ be the set of distinguished right coset representatives for $W_{1}^{d} \cap W_{2}$ in $W_{2}$. Then for any $t \in T_{d}$, we have

(1) $d t \in R$,

(2) $l(d t)=l(d)+l(t)$. 
Proof. (1) Suppose $d t \notin R$. Then $l\left(w_{1} d t\right)<l(d t)$ for some $w_{1} \in W_{1}$, so choose among all $w_{1} \in W_{1}$ with $l\left(w_{1} d t\right)<l(d t)$ an element $\hat{w}_{1}$ of minimal length. Write $\hat{w}_{1}=\tilde{w}_{1} v$ with $\tilde{w}_{1} v$ reduced and $\tilde{w}_{1}$ a fundamental reflection. Thus

$$
l\left(\hat{w}_{1} d t\right)=l\left(\tilde{w}_{1} v d t\right)<l(d t) \leq l(v d t) .
$$

Since $\tilde{w}_{1}$ is fundamental, Theorem (1.1.5) implies that one of three cases occurs:

$$
\tilde{w}_{1} v d t= \begin{cases}v^{\prime} d t, & \text { where } l\left(v^{\prime}\right)=l(v)-1 \\ v d^{\prime} t, & \text { if the exchange takes place in } v, \\ & \text { if the exchange takes place in } d, \\ v d t^{\prime}, & \text { where } l\left(t^{\prime}\right)=l(t)-1 \\ & \text { if the exchange takes place in } t .\end{cases}
$$

The first case cannot happen since $\tilde{w}_{1} v$ is reduced. If $\tilde{w}_{1} v d t=v d^{\prime} t$, then $d^{\prime}=v^{-1} \tilde{w}_{1} v d \in W_{1} d W_{2}$ which contradicts the fact that $d$ is distinguished. If $\tilde{w}_{1} v d t=v d t^{\prime}$, then $t^{\prime}=\left(d^{-1} v^{-1} \tilde{w}_{1} v d\right) t \in\left(W_{1}^{d} \cap W_{2}\right) t$, contradicting the fact that $t$ is distinguished. Therefore $d t \in R$.

(2) Since $t \in W_{2}$, this follows immediately from Lemma (2.17).

Lemma (2.22). Let $M$ be an $A$-A bimodule and $N \leq M$ an $A$ - $A_{1}$ bimodule. Let $W_{2}$ be any parabolic subgroup of $W$. Suppose $M \simeq N \otimes_{A_{1}} A$. Then $M$ may be decomposed into chunks that are $A_{2}$ right-stable: $M \simeq$ $\sum_{d \in W_{1} \backslash W / W_{2} \text {, distinguished }} N a_{d} A_{2}$.

(Note. This is also a decomposition into $A_{2}$ left-stable pieces since $N$ is a left $A$-module.)

Proof. Let $D$ be the set of distinguished $W_{1}-W_{2}$ double coset representatives in $W$ and let $R$ be the set of distinguished right coset representatives for $W_{1}$ in $W$. Decompose $W$ into $W_{1}-W_{2}$ double cosets:

$$
W=\bigcup_{d \in D} W_{1} d W_{2}
$$

then

$$
R=\bigcup_{d \in D}\left(R \cap W_{1} d W_{2}\right) .
$$

For a fixed $d \in D$, let $T_{d}$ be the set of distinguished right coset representatives for $W_{1}^{d} \cap W_{2}$ in $W_{2}$. Then $d T_{d} \leq R$ by Lemma (2.21). Also, $d T_{d} \leq W_{1} d W_{2}$ since $T_{d} \leq W_{2}$. Thus $d T_{d} \leq R \cap W_{1} d W_{2}$. Now let $r \in R \cap W_{1} d W_{2}$. Since $r \in W_{1} d W_{2}$, we may write it as $w_{1} d w_{2}$ where $w_{1} \in W_{1}, w_{2} \in W_{2}$, and $l\left(w_{1} d w_{2}\right)=l\left(w_{1}\right)+l(d)+l\left(w_{2}\right)$ by Proposition (1.3.2). Since $r \in R, r$ is distinguished in $W_{1} r=W_{1} w_{1} d w_{2}$, and so $l\left(w_{1}^{-1} w_{1} d w_{2}\right)=l\left(w_{1}^{-1}\right)+l\left(w_{1} d w_{2}\right)$ 
by Proposition (1.1.2). But $w_{1}^{-1} w_{1} d w_{2}=d w_{2}$ and $l\left(d w_{2}\right)=l(d)+l\left(w_{2}\right)$ by Lemma (2.17). Combining these facts we have

$$
\begin{aligned}
l(d)+l\left(w_{2}\right) & =l\left(d w_{2}\right)=l\left(w_{1}^{-1} w_{1} d w_{2}\right) \\
& =l\left(w_{1}^{-1}\right)+l\left(w_{1} d w_{2}\right)=l\left(w_{1}^{-1}\right)+l\left(w_{1}\right)+l(d)+l\left(w_{2}\right),
\end{aligned}
$$

which implies that $l\left(w_{1}\right)=0$. Hence $w_{1}$ is the identity and $r=d w_{2}$.

Suppose $w_{2} \notin T_{d}$. Then $l\left(d^{-1} w_{1} d w_{2}\right)<l\left(w_{2}\right)$ for some $d^{-1} w_{1} d \in W_{1}^{d} \cap$ $W_{2}$. Since $d^{-1} w_{1} d w_{2} \in W_{2}$, we have by Lemma (2.17) that $l\left(d\left(d^{-1} w_{1} d w_{2}\right)\right)=$ $l(d)+l\left(d^{-1} w_{1} d w_{2}\right)$. Thus

$$
l\left(w_{1} d w_{2}\right)=l\left(d\left(d^{-1} w_{1} d w_{2}\right)\right)<l(d)+l\left(w_{2}\right)=l\left(d w_{2}\right) .
$$

But $d\left(d^{-1} w_{1} d w_{2}\right)=w_{1} d w_{2} \in W_{1} d w_{2}$ and $d w_{2} \in R$. Hence $l\left(w_{1} d w_{2}\right)>$ $l\left(d w_{2}\right)$, which gives a contradiction. Therefore $r \in d T_{d}$ so that

$$
d T_{d}=R \cap W_{1} d W_{2}
$$

Thus

$$
\sum_{r \in R \cap W_{1} d W_{2}} N a_{r}=\sum_{t \in T_{d}}\left(N a_{d}\right) a_{t} .
$$

Since $\bigoplus_{t \in T_{d}}\left(A_{1} a_{d}\right) a_{t}=A_{1} a_{d} A_{2}$ (as in the proof of Corollary (2.20)), we have

$$
\sum_{t \in T_{d}}\left(N a_{d}\right) a_{t}=N a_{d} A_{2}
$$

which is $A_{2}$ right-stable. So

$$
N \otimes_{A_{1}} A=\sum_{r \in R} N a_{r}=\sum_{d \in D} \sum_{t \in T_{d}}\left(N a_{d}\right) a_{t}=\sum_{d \in D} N a_{d} A_{2} .
$$

Theorem (2.23). Let $M$ be an $A-A$ bimodule and $N$ an $A-A^{\prime}$ bisubmodule of $M$ such that $M \simeq N \otimes_{A^{\prime}} A$. Then $Z_{M}(A)=N_{W^{\prime}, W^{\prime}}\left(Z_{N}\left(A^{\prime}\right)\right)$.

Proof. By Proposition (2.13) we have that $N_{W, W^{\prime}}\left(Z_{M}\left(A^{\prime}\right)\right) \leq Z_{M}(A)$. Since $N \leq M$, it follows that $Z_{N}\left(A^{\prime}\right) \leq Z_{M}\left(A^{\prime}\right)$, and hence $N_{W, W^{\prime}}\left(Z_{N}\left(A^{\prime}\right)\right) \leq$ $N_{W, W^{\prime \prime}}\left(Z_{M}\left(A^{\prime}\right)\right) \leq Z_{M}(A)$. So we must show that $Z_{M}(A) \leq N_{W, W^{\prime}}\left(Z_{N}\left(A^{\prime}\right)\right)$. First we consider the case $W=\langle w\rangle$ where $w$ is a single fundamental reflection and $W^{\prime}=\{1\}$. Let $m \in Z_{M}(A)$. Since $M \simeq N \otimes_{A^{\prime}} A \simeq N \oplus N a_{w^{\prime}}$, write $m$ as $m=n_{0}+n_{1} a_{w}$, with $n_{0}, n_{1} \in N$. Since $a_{w} m=m a_{w}$, we get

$$
\begin{aligned}
a_{w}\left(n_{0} a_{1}+n_{1} a_{w}\right) & =\left(a_{w} n_{0}\right) a_{1}+\left(a_{w} n_{1}\right) a_{w} \\
& =\left(n_{0} a_{1}+n_{1} a_{w}\right) a_{w}=n_{0} a_{w}+n_{1} a_{w}^{2} \\
& =n_{0} a_{w}+n_{1}\left(u a_{1}+(u-1) a_{w}\right) \\
& =\left(u n_{1}\right) a_{1}+\left((u-1) n_{1}+n_{0}\right) a_{w} .
\end{aligned}
$$


Equating terms in $N$ gives $a_{w} n_{0}=u n_{1}$. Then $a_{w} n_{0} a_{w}=u n_{1} a_{w}$ and so $n_{1} a_{w}=u^{-1} a_{w} n_{0} a_{w}$. Thus

$$
m=n_{0}+u^{-1} a_{w} n_{0} a_{w}=N_{W, W^{\prime}}\left(n_{0}\right) \in N_{W, W^{\prime}}\left(Z_{N}\left(A^{\prime}\right)\right) .
$$

Now for the general situation write $N \otimes_{A^{\prime}} A \simeq \bigoplus_{r \in R} N a_{r}$ where $R$ is the set of distinguished right coset representatives for $W^{\prime}$ in $W$. Let $m \in Z_{M}(A)$. Write $m=n_{1}+\sum_{r \in R, r \neq 1} n_{r} a_{r}, n_{r} \in N$. We will show that $m \in N_{W, W^{\prime}}\left(Z_{N}\left(A^{\prime}\right)\right)$ by showing that $n_{1} \in Z_{N}\left(A^{\prime}\right)$ and $m=N_{W, W^{\prime}}\left(n_{1}\right)$.

Let $w \in W^{\prime}$ be a fundamental reflection. Then $a_{w} m=m a_{w}$ which gives

$$
\begin{aligned}
a_{w}\left(n_{1}+\sum_{\substack{r \in R \\
r \neq 1}} n_{r} a_{r}\right) & =a_{w} n_{1}+\sum_{\substack{r \in R \\
r \neq 1}} a_{w} n_{r} a_{r} \\
& =\left(n_{1}+\sum_{\substack{r \in R \\
r \neq 1}} n_{r} a_{r}\right) a_{w}=n_{1} a_{w}+\sum_{\substack{r \in R \\
r \neq 1}} n_{r} a_{r} a_{w} .
\end{aligned}
$$

Equating terms in $N$ we get that $a_{w} n_{1}=n_{1} a_{w}$. Thus $n_{1} \in Z_{N}\left(A^{\prime}\right)$.

Now let $r \in R$ be minimum in the sense that for all $\tilde{r} \in R$ with $l(\tilde{r})<l(r)$ we know that

$$
n_{\tilde{r}} a_{\grave{r}}=u^{-l(\tilde{r})} a_{\tilde{r}-1} n_{1} a_{\tilde{r}} .
$$

Let $w_{1} w_{2} \cdots w_{k}$ be a reduced expression for $r$ with the $w_{i}$ fundamental reflections. Suppose that $w_{1} w_{2} \cdots w_{k-1} \in W^{\prime} \hat{r}, \hat{r} \in R$. Then $w_{1} w_{2} \cdots w_{k-1}=w^{\prime} \hat{r}$ for some $w^{\prime} \in W^{\prime}$ and $l\left(w^{\prime} \hat{r}\right)=l\left(w^{\prime}\right)+l(\hat{r})$. But then $r=w_{1} w_{2} \cdots w_{k-1} w_{k}=$ $w^{\prime} \hat{r} w_{k}$ with $l\left(w^{\prime} \hat{r} w_{k}\right)=l\left(w^{\prime}\right)+l(\hat{r})+l\left(w_{k}\right)$ and $w^{\prime-1}\left(w^{\prime} \hat{r} w_{k}\right)$, whose length is $l(\hat{r})+l\left(w_{k}\right)$, is an element in $W^{\prime} r$. Hence, since $r$ is distinguished in $W r$, it must be that $l\left(w^{\prime}\right)=0$ and so $w^{\prime}=1$. That is, $w_{1} w_{2} \cdots w_{k-1}=\hat{r}$. Let $W_{k}=\left\langle w_{k}\right\rangle$. Note that $\hat{r}$ is distinguished in $W^{\prime} \hat{r} W_{k}$ in $W$. Also $\left(W^{\prime}\right)^{\hat{r}} \cap W_{k}$ is trivial. For if $w_{k} \in \hat{r}^{-1} W^{\prime} \hat{r}$, then $w_{k}=\hat{r}^{-1} w^{\prime} \hat{r}$ for some $w^{\prime} \in W^{\prime}$ which would imply that $r=w^{\prime} \hat{r} \in W^{\prime} \hat{r}$, a contradiction. Letting $V_{1}=N a_{\hat{r}}+\left(N a_{\hat{r}}\right) a_{w_{k}}$ and $V_{2}=\sum_{d \in W^{\prime} \backslash W / W_{k}}$, distinguished; $d \neq \hat{r} N a_{d} A\left(W_{k}\right)$, Lemma (2.22) implies that

$$
N \otimes_{A^{\prime}} A=V_{1}+V_{2}
$$

is a decomposition into $A\left(W_{k}\right)$-bimodules. Thus

$$
Z_{M}\left(A\left(W_{k}\right)\right)=Z_{V_{1}}\left(A\left(W_{k}\right)\right)+Z_{V_{2}}\left(A\left(W_{k}\right)\right)
$$

and since $m \in Z_{M}(A)$, we have that $n_{\hat{r}} a_{\hat{r}}+\left(n_{r} a_{\hat{r}}\right) a_{w_{k}} \in Z_{V_{1}}\left(A\left(W_{k}\right)\right)$. Thus we may apply the result of this theorem to $V_{1}$ since this is just the special case of a single fundamental reflection whose proof was given early in the proof of this theorem. This gives us

$$
n_{r} a_{r}=\left(n_{r} a_{\hat{r}}\right) a_{w_{k}}=u^{-1} a_{w_{k}} n_{\hat{r}} a_{\hat{r}} a_{w_{k}} .
$$


By the minimality of $r$, we have

$$
n_{\hat{r}} a_{\hat{r}}=u^{-l(\hat{r})} a_{\hat{r}-1} n_{1} a_{\hat{r}}
$$

Hence

$$
n_{r} a_{r}=u^{-1} a_{w_{k}}\left(u^{-l(\hat{r})} a_{\hat{r}^{-1}} n_{1} a_{\hat{r}}\right) a_{w_{k}}=u^{-l(r)} a_{r^{-1}} n_{1} a_{r}
$$

after all. Therefore

$$
m=\sum_{r \in R} u^{-l(r)} a_{r^{-1}} n_{1} a_{r}=N_{W, W^{\prime}}\left(n_{1}\right),
$$

and the theorem is proved.

Corollary (2.24). Let $M$ be an $A-A$ bimodule and $N$ an $A-A^{\prime}$ bisubmodule of $M$ such that $M \simeq N \otimes_{A^{\prime}} A$. Then

$$
Z_{M}(A)=N_{W, W^{\prime}}\left(Z_{M}\left(A^{\prime}\right)\right) .
$$

Proof. By Proposition (2.13), we have $N_{W, W^{\prime}}\left(Z_{M}\left(A^{\prime}\right)\right) \leq Z_{M}(A)$. Since $N \leq$ $M$, we have

$$
N_{W, W^{\prime}}\left(Z_{N}(A)\right) \leq N_{W, W^{\prime}}\left(Z_{M}\left(A^{\prime}\right)\right) .
$$

Theorem (2.23) then implies that

$$
Z_{M}(A)=N_{W, W^{\prime}}\left(Z_{N}\left(A^{\prime}\right)\right) \leq N_{W, W^{\prime}}\left(Z_{M}\left(A^{\prime}\right)\right) \leq Z_{M}(A)
$$

and the corollary follows.

Corollary (2.25). Let $S$ be a direct summand of an $A$ - $A$ bimodule $M=N \otimes_{A^{\prime}} A$ with $N$ an $A-A^{\prime}$ bimodule. Then

$$
N_{W, W^{\prime}}\left(Z_{S}\left(A^{\prime}\right)\right)=Z_{S}(A) .
$$

Proof. Write $M=S \oplus(M-S)$. Theorem (2.23) implies that

$$
N_{W, W^{\prime}}\left(Z_{M}\left(A^{\prime}\right)\right)=Z_{M}(A) .
$$

From Proposition (2.13) we get

$$
N_{W^{\prime}, W^{\prime}}\left(Z_{S}\left(A^{\prime}\right)\right) \leq Z_{S}(A) \text {. }
$$

Let $s \in Z_{S}(A)$. Then $s \in Z_{M}(A)=N_{W, W^{\prime}}\left(Z_{M}\left(A^{\prime}\right)\right)$. Write

$$
s=\sum_{r \in R} u^{-l(r)} a_{r^{-1}} m a_{r}
$$

for some $m \in Z_{M}\left(A^{\prime}\right)$, where $R$ is the set of distinguished right coset representatives for $W^{\prime}$ in $W$. Decompose $m$ as $\hat{s}+t$ where $\hat{s} \in S$ and $t \in M-S$. Then

$$
s=\sum_{r \in R} u^{-l(r)} a_{r^{-1}} \hat{s} a_{r}+\sum_{r \in R} u^{-l(r)} a_{r^{-1}} t a_{r}
$$


and

$$
s-\sum_{r \in R} u^{-l(r)} a_{r^{-1}} \hat{s} a_{r} \in S
$$

But

$$
\sum_{r \in R} u^{-l(r)} a_{r^{-1}} t a_{r} \in M-S
$$

Thus

$$
s-\sum_{r \in R} u^{-l(r)} a_{r^{-1}} \hat{s} a_{r} \in S \cap(M-S)=\{0\} .
$$

Hence

$$
s=\sum_{r \in R} u^{-l(r)} a_{r^{-1}} \hat{s} a_{r} \in N_{W, W^{\prime}}\left(Z_{S}\left(A^{\prime}\right)\right)
$$

from which the corollary is deduced.

Theorem (2.26). Let $W_{1}$ and $W_{2}$ be parabolic subgroups of $W$ such that $W_{2}=$ $W_{1}^{w}$ for some $w \in W$. Let $M$ be an $A-A$ bimodule and $N$ an $A-A_{1}$ bisubmodule of $M$ such that $M=N \otimes_{A_{1}} A$. Then there is an $A-A_{2}$ bisubmodule $N^{\prime}$ of $M$ such that

$$
N_{W, W_{1}}\left(Z_{N}\left(A_{1}\right)\right)=N_{W, W_{2}}\left(Z_{N^{\prime}}\left(A_{2}\right)\right) .
$$

Proof. Let $d$ be distinguished in the double coset $W_{1} w W_{2}$ in $W$. Let $N^{\prime}$ be the $A-A_{2}$ bimodule $N \otimes_{A_{1}} a_{d}$. By Lemma (2.15) we have that $M \simeq N^{\prime} \otimes_{A_{2}} A$. Applying Theorem (2.23) gives

$$
N_{W, W_{2}}\left(Z_{N^{\prime}}\left(A_{2}\right)\right)=Z_{M}(A) \text {. }
$$

But also we may apply Theorem (2.23) to $M=N \otimes_{A_{1}} A$ to get

$$
N_{W, W_{1}}\left(Z_{N}\left(A_{1}\right)\right)=Z_{M}(A) .
$$

This proves the result.

Corollary (2.27). Given the same hypotheses as Theorem (2.26), we can conclude that

$$
N_{W, W_{1}}\left(Z_{M}\left(A_{1}\right)\right)=N_{W, W_{2}}\left(Z_{M}\left(A_{2}\right)\right) .
$$

Proof. Applying Corollary (2.26) to $N \otimes_{A_{1}} A$ we have

$$
Z_{M}(A)=N_{W, W_{1}}\left(Z_{M}\left(A_{1}\right)\right) \text {. }
$$

From the proof of Theorem (2.26) we know that $M \simeq\left(N \otimes_{A_{1}} a_{d}\right) \otimes_{A_{2}} A$ where $d \in W_{1} w W_{2}$ is distinguished. Applying Corollary (2.24) again gives

$$
Z_{M}(A)=N_{W, W_{2}}\left(Z_{M}\left(A_{2}\right)\right) \text {. }
$$

Lemma (2.28). Let $W_{1}$ and $W_{2}$ be parabolic subgroups of $W$ and let $D$ be the set of distinguished $W_{1}-W_{2}$ double coset representatives in $W$. Then

$$
A=\bigoplus_{d \in D} A_{1} a_{d} A_{2} .
$$

Proof. This follows immediately from the decomposition $W=\dot{U}_{d \in D} W_{1} d W_{2}$. 
Theorem (2.29) (Mackey decomposition). Let $W_{1}$ and $W_{2}$ be parabolic subgroups of $W$ and let $D$ be the set of distinguished $W_{1}-W_{2}$ double coset representatives in $W$. Also, let $N$ be an $A-A_{1}$ bimodule. Then

$$
N \otimes_{A_{1}} A \simeq \bigoplus_{d \in D}\left(\left(N \otimes_{A_{1}} a_{d}\right) \otimes_{A\left(W_{1}^{d} \cap W_{2}\right)} A_{2}\right) .
$$

Proof. From Lemma (2.28) we have $A=\bigoplus_{d \in D} A_{1} a_{d} A_{2}$. By Corollary (2.20), $A_{1} a_{d} A_{2} \simeq A_{1} a_{d} \otimes_{A\left(W_{1}^{d} \cap W_{2}\right)} A_{2}$. Thus

$$
A \simeq \bigoplus_{d \in D}\left(A_{1} a_{d} \otimes_{A\left(W_{1}^{d} \cap W_{2}\right)} A_{2}\right)
$$

and hence

$$
\begin{aligned}
N \otimes_{A_{1}} A & \simeq N \otimes_{A_{1}}\left(\bigoplus_{d \in D} A_{1} a_{d} \otimes_{A\left(W_{1}^{d} \cap W_{2}\right)} A_{2}\right) \\
& \simeq \bigoplus_{d \in D}\left(\left(N \otimes_{A_{1}} A_{1} a_{d}\right) \otimes_{A\left(W_{1}^{d} \cap W_{2}\right)} A_{2}\right) \\
& \simeq \bigoplus_{d \in D}\left(\left(N \otimes_{A_{1}} a_{d}\right) \otimes_{A\left(W_{1}^{d} \cap W_{2}\right)} A_{2}\right)
\end{aligned}
$$

Theorem (2.30). Let $W_{1}$ and $W_{2}$ be parabolic subgroups of $W$. Let $R$ be the set of distinguished right coset representatives for $W_{1}$ in $W$. Let $D$ be the set of distinguished $W_{1}-W_{2}$ double coset representatives in $W$. For $d \in D$, let $T_{d}$ be the set of distinguished right coset representatives for $W_{1}^{d} \cap W_{2}$ in $W_{2}$. Let $M$ be an $A$ - $A$ bimodule and let $b \in Z_{M}\left(A_{1}\right)$. Then

(1) for $d \in D, u^{-l(d)} a_{d^{-1}} b a_{d} \in Z_{M}\left(A\left(W_{1}^{d} \cap W_{2}\right)\right)$.

(2) $N_{W, W_{1}}(b)=\sum_{d \in D} N_{W_{2}, W_{1}^{d} \cap W_{2}}\left(u^{-l(d)} a_{d^{-1}} b a_{d}\right)$.

Proof. (1) Let $w \in W_{1}^{d} \cap W_{2}$ be a fundamental reflection. Then $w=d^{-1} w_{1} d$ for some $w_{1} \in W_{1}$. In other words, $d w=w_{1} d$ and $l(d)+l(w)=l(d w)=$ $l\left(w_{1} d\right)=l\left(w_{1}\right)+l(d)$ by Lemma (2.17). Thus $l\left(w_{1}\right)=1$ and so $w_{1}$ is fundamental. Hence,

$$
\begin{aligned}
l\left(d^{-1} w_{1}\right) & =l\left(\left(d^{-1} w_{1}\right)^{-1}\right)=l\left(w_{1}^{-1} d\right) \\
& =l\left(w_{1} d\right)=l\left(w_{1}\right)+l(d)=l\left(w_{1}\right)+l\left(d^{-1}\right) .
\end{aligned}
$$

Similarly, $l\left(w d^{-1}\right)=l(w)+l\left(d^{-1}\right)$. Therefore

$$
\begin{aligned}
\left(a_{d^{-1}} b a_{d}\right) a_{w} & =a_{d^{-1}} b a_{d w}=a_{d^{-1}} b a_{w_{1} d} \\
& =a_{d^{-1}} b a_{w_{1}} a_{d}=a_{d^{-1}} a_{w_{1}} b a_{d} \\
& =a_{d^{-1} w_{1}} b a_{d}=a_{w d^{-1}} b a_{d}=a_{w}\left(a_{d^{-1}} b a_{d}\right) .
\end{aligned}
$$


(2) Let $d \in D$ and $t \in T_{d}$. By Lemma (2.21), $d t \in R$ and $l(d t)=l(d)+l(t)$. Thus

$$
\begin{aligned}
\sum_{d \in D} & N_{W_{2}, W_{1}^{d} \cap W_{2}}\left(u^{-l(d)} a_{d^{-1}} b a_{d}\right) \\
& =\sum_{d \in D} \sum_{t \in T_{d}} u^{-l(t)} a_{t^{-1}}\left(u^{-l(d)} a_{d^{-1}} b a_{d}\right) a_{t} \\
& =\sum_{d \in D} \sum_{t \in T_{d}} u^{-(l(t)+l(d))} a_{t^{-1}} a_{d^{-1}} b a_{d} a_{t} \\
& =\sum_{d \in D} \sum_{t \in T_{d}} u^{-l(d t)} a_{(d t)^{-1}} b a_{d t} \\
& =\sum_{r \in R} u^{-l(r)} a_{r^{-1}} b a_{r} \quad \text { since } \sum_{d \in D}\left|W_{1} d W_{2}\right|=|W| \\
& =N_{W, W_{1}}(b) . \quad \text { and }\left|W_{1} d W_{2}\right|=\left|W_{1}\right| \cdot\left[W_{2}: W_{1}^{d} \cap W_{2}\right]
\end{aligned}
$$

Corollary (2.31). Let $M$ be an A-algebra (i.e., the action on $M$ arises from an $R$-algebra homomorphism $A \rightarrow M)$. Let $W_{1}, W_{2}$ and $D$ be as in Theorem (2.30). Then

$$
N_{W, W_{1}}\left(Z_{M}\left(A_{1}\right)\right) \cdot N_{W, W_{2}}\left(Z_{M}\left(A_{2}\right)\right) \leq \sum_{d \in D} N_{W, W_{1}^{d} \cap W_{2}}\left(Z_{M}\left(A\left(W_{1}^{d} \cap W_{2}\right)\right)\right) .
$$

Proof. Let $m_{1} \in Z_{M}\left(A_{1}\right)$ and $m_{2} \in Z_{M}\left(A_{2}\right)$. Then

$$
\begin{aligned}
N_{W, W_{1}} & \left(m_{1}\right) \cdot N_{W, W_{2}}\left(m_{2}\right) \\
& =N_{W, W_{2}}\left(N_{W, W_{1}}\left(m_{1}\right) m_{2}\right) \quad \text { by Proposition }(2.13) \\
& =N_{W^{\prime}, W_{2}}\left(\sum_{d \in D} N_{W_{2}, W_{1}^{d} \cap W_{2}}\left(u^{-l(d)} a_{d^{-1}} m_{1} a_{d}\right) m_{2}\right) \quad \text { by Theorem }(2.30) \\
& =N_{W, W_{2}}\left(\sum_{d \in D} N_{W_{2}, W_{1}^{d} \cap W_{2}}\left(u^{-l(d)} a_{d^{-1}} m_{1} a_{d} \cdot m_{2}\right)\right) \text { since } m_{2} \in Z_{M}\left(A_{2}\right) \\
& =\sum_{d \in D} N_{W, W_{1}^{d} \cap W_{2}}\left(u^{-l(d)} a_{d^{-1}} m_{1} a_{d} \cdot m_{2}\right) \quad \text { by Lemma }(2.12)
\end{aligned}
$$

which proves the corollary since $u^{-l(d)} a_{d^{-1}} m_{1} a_{d} \cdot m_{2} \in Z_{M}\left(A\left(W_{1}^{d} \cap W_{2}\right)\right)$.

Proposition (2.32). Let $W_{1}$ and $W_{2}$ be parabolic subgroups of $W$. Let $\widehat{W}$ be a parabolic subgroup of $W$ contained in $W_{1} \times W_{2}$ so that $\widehat{W}=\widehat{W}_{1} \times \widehat{W}_{2}$ for some parabolic subgroups $\widehat{W}_{1}$ of $W_{1}$ and $\widehat{W}_{2}$ of $W_{2}$. Let $\hat{w} \in \widehat{W}$. Then

$$
N_{W_{1} \times u_{2}, \widehat{W}^{\prime}}\left(a_{\hat{u}}\right)=N_{W_{1}, \widehat{w}_{1}}\left(a_{\hat{u}_{1}}\right) \cdot N_{w_{2}, W_{2}}\left(a_{\hat{u}_{2}}\right),
$$

where $\hat{w}=\hat{w}_{1} \hat{w}_{2}$ with $\hat{w}_{1} \in \widehat{W}_{1}, \hat{w}_{2} \in \widehat{W}_{2}$, and of course $l\left(\hat{w}_{1} \hat{w}_{2}\right)=l\left(\hat{w}_{1}\right)+$ $l\left(\hat{w}_{2}\right)$. 
Proof. Clearly we may factor $\hat{w}$ as described in the statement of the proposition. Thus

$$
\begin{aligned}
N_{W_{1} \times W_{2}, \widehat{W}}\left(a_{\hat{w}}\right) & =N_{W_{1} \times W_{2}, \widehat{W}_{1} \times \widehat{W}_{2}}\left(a_{\hat{w}_{1} \hat{w}_{2}}\right) \\
& =N_{W_{1} \times W_{2}, W_{1} \times \widehat{W}_{2}}\left(N_{W_{1} \times \widehat{W}_{2}, \widehat{W}_{1} \times \widehat{W}_{2}}\left(a_{\hat{w}_{1} \hat{w}_{2}}\right)\right) \quad \text { by Lernma }(2.12) \\
& =N_{W_{2}, \widehat{W}_{2}}\left(N_{W_{1}, \widehat{W}_{1}}\left(a_{\hat{w}_{1} \hat{w}_{2}}\right)\right) \\
& =N_{W_{2}, \widehat{W}_{2}}\left(N_{W_{1}, \widehat{W}_{1}}\left(a_{\hat{w}_{1}}\right) a_{\hat{w}_{2}}\right) \\
& =N_{W_{1}, \widehat{W}_{1}}\left(a_{\hat{w}_{1}}\right) \cdot N_{W_{2}, \widehat{W}_{2}}\left(a_{\hat{w}_{2}}\right) .
\end{aligned}
$$

Definition (2.33). A right $A$-module $M$ is $A^{\prime}$-projective if every exact diagram of right $A$-modules and maps which can be completed to a commutative diagram over $A^{\prime}$ can also be completed to a commutative diagram over $A$ :

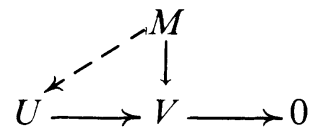

Theorem (2.34) ("Higman's Criterion" for $A$-modules). Let $M$ be a right $A$ module. Then the following are equivalent:

(1) $M$ is $A^{\prime}$-projective.

(2) $M \mid M \otimes_{A^{\prime}} A$; that is, $M$ is isomorphic to a direct summand of $M \otimes_{A^{\prime}} A$.

(3) $M \mid U \otimes_{A^{\prime}} A$ for some right $A^{\prime}$-module $U$.

(4) $M_{W, W^{\prime}}\left(\operatorname{Hom}_{A^{\prime}}(M, M)\right)=\operatorname{Hom}_{A}(M, M)$.

Proof. (1) $\Rightarrow(2)$ Let $f: M \otimes_{A^{\prime}} A \rightarrow M$ be defined as $f(m \otimes a)=m a$. This is surjective and we can complete the following diagram to make it a commutative $A^{\prime}$-diagram:

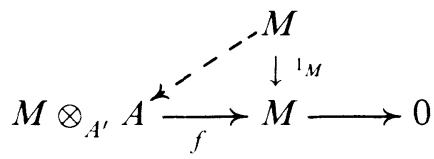

So by (1) this can be completed to a commutative diagram over $A$, say by $\hat{\theta}$. Thus $f \hat{\theta}=1_{M}$ and $M \mid M \otimes_{A^{\prime}} A$.

(2) $\Rightarrow(3)$ This is obvious.

(3) $\Rightarrow$ (4) Define $g: U \otimes_{A^{\prime}} A \rightarrow U \otimes_{A^{\prime}} A$ by

$$
\begin{aligned}
& \left(\sum_{i \in R} u_{i} \otimes a_{i}\right) g=u_{1} \otimes a_{1} ; \\
R= & \text { set of distinguished right coset } \\
& \text { representatives for } W^{\prime} \text { in } W .
\end{aligned}
$$


Then for $a^{\prime} \in A^{\prime}$,

$$
\begin{aligned}
\left(\left(\sum_{i \in R} u_{i} \otimes a_{i}\right) a^{\prime}\right) g & =\left(\sum_{i \in R} u_{i} \otimes a_{i} a^{\prime}\right) g \\
& =u_{1} \otimes a^{\prime}=\left(u_{1} \otimes a_{1}\right) a^{\prime}=\left(\sum_{i \in R} u_{i} \otimes a_{i}\right) g \cdot a^{\prime}
\end{aligned}
$$

So that $g$ is an $A^{\prime}$-map.

Let $v=\sum_{i \in R} u_{i} \otimes a_{i} \in U \otimes_{A^{\prime}} A$. Then

$$
\begin{aligned}
\left(\sum_{i \in R} u_{i} \otimes a_{i}\right) N_{W, W^{\prime}}(g) & =\left(\sum_{i \in R} u_{i} \otimes a_{i}\right)\left(\sum_{r \in R} u^{-l(r)} a_{r^{-1}} g a_{r}\right) \\
& =\sum_{r \in R}\left(u_{r} \otimes a_{1}\right) a_{r}=\sum_{r \in R}\left(u_{r} \otimes a_{r}\right)=v
\end{aligned}
$$

so that $N_{W, W^{\prime}}(g)=1_{U \otimes_{A^{\prime}} A}=$ identity on $U \otimes_{A^{\prime}} A$.

Let $h$ be the projection of $U \otimes_{A^{\prime}} A$ onto $M$. Clearly $h$ is an $A$-map. Thus the restriction of $g h$ to $M$, denoted $\left.g h\right|_{M}$, is an element of $\operatorname{Hom}_{A^{\prime}}(M, M)$. Then for $m \in M$,

$$
(m) N_{W, W^{\prime}}\left(\left.g h\right|_{M}\right)=m,
$$

and so

$$
1_{M}=N_{W, W^{\prime}}\left(\left.g h\right|_{M}\right) \in N_{W, W^{\prime}}\left(\operatorname{Hom}_{A^{\prime}}(M, M)\right)
$$

which implies (4).

(4) $\Rightarrow(1)$ There exists $\psi \in \operatorname{Hom}_{A^{\prime}}(M, M)$ such that $N_{W, W^{\prime}}(\psi)=1_{M}$. Assume the following exact diagram of right $A$-modules can be completed by $\theta$ to a commutative diagram over $A^{\prime}$ :

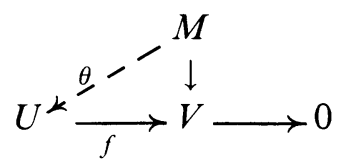

Then

$$
N_{W, W^{\prime}}(\psi \theta) f=N_{W, W^{\prime}}(\psi \theta f)=N_{W, W^{\prime}}(\psi g)=N_{W, W^{\prime}}(\psi) g=g .
$$

Since $N_{W, W^{\prime}}(\psi \theta)$ is an $A$-map by Proposition (2.13), (1) follows and this completes the proof of the theorem.

Remark. Theorem (2.34) also holds on either side of an $A-A$ bimodule $M$.

Theorem (2.35). Let $Z$ be a finitely generated indecomposable right A-module. Then there exists a parabolic subgroup $W^{\prime}$ of $W$ unique up to conjugation such that $Z$ is $A^{\prime}$-projective and such that $W^{\prime}$ is $W$-conjugate to a parabolic subgroup of any parabolic subgroup $W^{\prime \prime}$ of $W$ for which $Z$ is $A^{\prime \prime}$-projective.

Proof. Let $V(Z)$ be the set of all subgroups $\widehat{W}$ of $W$ for which $Z$ is $A(\widehat{W})$ projective. Let $W^{\prime}$ be a minimal element of $V(Z)$ with respect to the partial order containment. Let $W^{\prime \prime}$ be any element of $V(Z)$. Write $Z=\bigoplus_{i=1}^{t} U_{i}$ 
with each $U_{i}$ an indecomposable right $A^{\prime}$-module. Then $Z \mid Z \otimes_{A^{\prime}} A$ $=\bigoplus_{i=1}^{t}\left(U_{i} \otimes_{A^{\prime}} A\right)$ and $Z \mid U_{i} \otimes_{A^{\prime}} A$ for some $i$ by the Krull-Schmidt Theorem [9] (with suitable hypotheses on $R$ ). We also have that $Z \mid Z \otimes_{A^{\prime \prime}} A$. Theorem (2.29) implies that

$$
Z \otimes_{A^{\prime \prime}} A=\bigoplus_{d \in D}\left(\left(Z \otimes_{A^{\prime \prime}} a_{d}\right) \otimes_{A\left(\left(W^{\prime \prime}\right)^{d} \cap W^{\prime}\right)} A^{\prime}\right),
$$

where $D$ is the set of distinguished $W^{\prime \prime}-W^{\prime}$ double coset representatives in $W$. Thus $Z \mid\left(Z \otimes_{A^{\prime \prime}} a_{d}\right) \otimes_{A\left(\left(W^{\prime \prime}\right) d^{d} \cap W^{\prime}\right)} A^{\prime}$ for some $d$ and so

$$
U_{i} \mid\left(Z \otimes_{A^{\prime \prime}} a_{d}\right) \otimes_{A\left(\left(W^{\prime \prime}\right) d^{d} \cap W^{\prime}\right)} A^{\prime}
$$

for this $d$. Then $U_{i} \otimes_{A^{\prime}} A \mid\left(Z \otimes_{A^{\prime \prime}} a_{d}\right) \otimes_{A\left(\left(W^{\prime \prime}\right)^{d} \cap W^{\prime}\right)} A$, and hence

$$
Z \mid\left(Z \otimes_{A^{\prime \prime}} a_{d}\right) \otimes_{\left.A\left(\left(W^{\prime \prime}\right)\right)^{d} \cap W^{\prime}\right)} A .
$$

That is, $Z$ is $A\left(\left(W^{\prime \prime}\right)^{d} \cap W^{\prime}\right)$-projective. But $W^{\prime}$ is minimal, so $W^{\prime} \leq\left(W^{\prime \prime}\right)^{d}$. Thus $\left(W^{\prime}\right)^{d^{-1}} \leq W^{\prime \prime}$, which proves the theorem.

\section{A BASIS FOR $Z\left(A\left(S_{n}\right)\right)$ OVER $\mathbf{Q}\left[u, u^{-1}\right]$}

Recall from $\S 1$ that $S_{n}$ is the Weyl group for SL $(n, q)$ and that $S_{n}$ is generated by the set $\{(12),(23), \ldots,(n-1 n)\}$ of fundamental reflections. In this section we obtain a basis for the center of $A\left(S_{n}\right)$ over $\mathbf{Q}\left[u, u^{-1}\right]$. This basis consists of relative norms of elements in certain parabolic subgroups of $S_{n}$, normed up from these subgroups to $S_{n}$. A particular element in $A\left(S_{n}\right)$ of special importance to this construction is given in the following definition.

Definition (3.1). The element $\eta$ in $A\left(S_{n}\right)$ is defined as

$$
\eta=N_{S_{n-1}, S_{1}}\left(a_{(12)(23) \cdots(n-1 n)}\right),
$$

with $S_{n-1}=\langle(12),(23), \ldots,(n-2 n-1)\rangle$.

Note. For $r \leq n-2, S_{n-r}$ will mean $\langle(12),(23), \ldots,(n-r-1 n-r)\rangle$, unless otherwise noted.

Lemma (3.2). $\eta \in Z\left(A\left(S_{n}\right)\right)$.

Proof. For $n=2$ this is clearly true. Proceed by induction. By Proposition (3.13) we have immediately that $\eta \in Z_{A\left(S_{n}\right)}\left(A\left(S_{n-1}\right)\right)$, so to prove this lemma, it will be enough to show that

$$
a_{(n-1 n)} \eta=\eta a_{(n-1 n)} .
$$

To do this, we will examine in further detail the element $\eta$. Let

$$
\alpha=N_{S_{n-2}, S_{1}}\left(a_{(12)(23) \cdots(n-2 n-1)}\right) .
$$


Then

$$
\begin{array}{rlrl}
\eta & =N_{S_{n-1}, S_{n-2}}\left(N_{S_{n-2}, S_{1}}\left(a_{(12)(23) \cdots(n-1 n)}\right)\right), \quad \text { by Lemma }(2.12) \\
& =N_{S_{n-1}, S_{n-2}}\left(\alpha a_{(n-1 n)}\right), & \text { since } a_{(n-1 n)} \in Z_{A\left(S_{n}\right)}\left(A\left(S_{n-2}\right)\right) \\
& =\alpha N_{S_{n-1}, S_{n-2}}\left(a_{(n-1 n)}\right), & & \text { by induction, } \alpha \in Z\left(A\left(S_{n-1}\right)\right) \\
& =N_{S_{n-1}, S_{n-2}}\left(a_{(n-1 n)}\right) \alpha, & & \text { by Proposition }(2.13), \\
& N_{S_{n-1}, S_{n-2}}\left(a_{(n-1 n)}\right) \in Z_{A\left(S_{n}\right)}\left(A\left(S_{n-1}\right)\right) .
\end{array}
$$

Hence, (3.3) holds if and only if

$$
a_{(n-1 n)} \alpha N_{S_{n-1}, S_{n-2}}\left(a_{(n-1 n)}\right)=N_{S_{n-1}, S_{n-2}}\left(a_{(n-1 n)}\right) \alpha a_{(n-1 n)} .
$$

From Theorem (2.30), part (2), with $W=S_{n-1}$ and $W_{1}=W_{2}=S_{n-2}$, we have that

$$
N_{S_{n-1}, S_{n-2}}\left(a_{(n-1 n)}\right)=a_{(n-1 n)}+u^{-1} N_{S_{n-2}, S_{n-3}}\left(a_{(n-2 n-1)(n-1 n)(n-2 n-1)}\right) .
$$

Replacing $N_{S_{n-1}, S_{n-2}}\left(a_{(n-1 n)}\right)$ with this expression in equation (3.4), we get a common term of $a_{(n-1 n)} \alpha a_{(n-1 n)}$ on both sides of the equation. Thus, by subtracting this common term and multiplying both sides by $u$, we have that (3.4) holds if and only if

$$
\begin{aligned}
& a_{(n-1 n)} \alpha N_{S_{n-2}, S_{n-3}}\left(a_{(n-2 n-1)(n-1 n)(n-2 n-1)}\right) \\
& \quad=N_{S_{n-2}, S_{n-3}}\left(a_{(n-2 n-1)(n-1 n)(n-2 n-1)}\right) \alpha a_{(n-1 n)} .
\end{aligned}
$$

Rewriting $a_{(n-2 n-1)(n-1 n)(n-2 n-1)}$ as $a_{(n-1 n)(n-2 n-1)(n-1 n)}$ using the relations in $S_{n}$ and recalling that $a_{(n-1 n)} \in Z_{A\left(S_{n}\right)}\left(A\left(S_{n-2}\right)\right)$ gives us that equation (3.5) is true if and only if

$$
\begin{aligned}
& a_{(n-1 n)} \alpha a_{(n-1 n)} N_{S_{n-2}, S_{n-3}}\left(a_{(n-2 n-1)}\right) a_{(n-1 n)} \\
& \quad=a_{(n-1 n)} N_{S_{n-2}, S_{n-3}}\left(a_{(n-2 n-1)}\right) a_{(n-1 n)} \alpha a_{(n-1 n)} .
\end{aligned}
$$

Now using Lemma (2.12) and the fact that $a_{(n-2 n-1)} \in Z_{A\left(S_{n-1}\right)}\left(A\left(S_{n-3}\right)\right)$, rewrite $\alpha$ as

$$
N_{S_{n-2}, S_{n-3}}\left(N_{S_{n-3}, S_{1}}\left(a_{(12)(23) \cdots(n-3 n-2)}\right) a_{(n-2 n-1)}\right) .
$$

By induction, $N_{S_{n-3}, S_{1}}\left(a_{(12)(23) \cdots(n-3 n-2)}\right) \in Z\left(A\left(S_{n-2}\right)\right)$, and so

$$
\alpha=N_{S_{n-3}, S_{1}}\left(a_{(12)(23) \cdots(n-3 n-2)}\right) \cdot N_{S_{n-2}, S_{n-3}}\left(a_{(n-2 n-1)}\right) .
$$

This expression for $\alpha$ allows us to rewrite the left-hand side of equation (3.6) as

$$
\begin{aligned}
a_{(n-1 n)} & N_{S_{n-3}, S_{1}}\left(a_{(12)(23) \cdots(n-3 n-2)}\right) \\
\cdot & N_{S_{n-2}, S_{n-3}}\left(a_{(n-2 n-1)}\right) a_{(n-1 n)} \\
\cdot & N_{S_{n-2}, S_{n-3}}\left(a_{(n-2 n-1)}\right) a_{(n-1 n)}
\end{aligned} .
$$


But $N_{S_{n-3}, S_{1}}\left(a_{(12)(23) \cdots(n-3 n-2)}\right) \in A\left(S_{n-2}\right)$ and by Proposition (2.13),

$$
N_{S_{n-2}, S_{n-3}}\left(a_{(n-2 n-1)}\right) \in Z_{A\left(S_{n-1}\right)}\left(A\left(S_{n-2}\right)\right) .
$$

Thus, recalling once again that $a_{(n-1 n)} \in Z_{A\left(S_{n}\right)}\left(A\left(S_{n-2}\right)\right)$, (3.7) may be rearranged to get

$$
\begin{aligned}
& a_{(n-1 n)} N_{S_{n-2}, S_{n-3}}\left(a_{(n-2 n-1)}\right) a_{(n-1 n)} \\
& \cdot N_{S_{n-3}, S_{1}}\left(a_{(12)(23) \cdots(n-3 n-2)}\right) N_{S_{n-2}, S_{n-3}}\left(a_{(n-2 n-1)}\right) a_{(n-1 n)} \\
& \quad=a_{(n-1 n)} N_{S_{n-2}, S_{n-3}}\left(a_{(n-2 n-1)}\right) a_{(n-1 n)} \alpha a_{(n-1 n)}
\end{aligned}
$$

which is the right-hand side of equations (3.6), and the lemma is proved.

Remark. We would like to be able to define $\eta$ as $N_{S_{n},\langle(12 \cdots n)\rangle}\left(a_{(12)(23) \cdots(n-1 n)}\right)$ to avoid the need for Lemma (3.2), but this is not permitted under our definition if the norm since $\langle(12 \cdots n)\rangle$ is not a parabolic subgroup of $S_{n}$. Nevertheless, $\langle(12 \cdots n)\rangle$ is, in some sense, close to being a parabolic subgroup. Since $S_{n}=\langle(12 \cdots n)\rangle S_{n-1}$, we see that $\langle(12 \cdots n)\rangle$ is like the difference of the two parabolic subgroups $S_{n}$ and $S_{n-1}$. Because of this, one might label $\langle(12 \cdots n)\rangle$ a virtual parabolic subgroup.

It will prove convenient to have various representations for the element $\eta$. In the next lemma we give some useful forms of $\eta$.

Lemma (3.8). The following are equal:

(1) $\eta$;

(2) $N_{S_{n-1}, S_{n-2}}\left(N_{S_{n-2}, S_{1}}\left(a_{(12)(23) \cdots(n-2 n-1)}\right) a_{(n-1 n)}\right)$;

(3) $N_{S_{n-2}, S_{1}}\left(a_{(12)(23) \cdots(n-2 n-1)}\right) N_{S_{n-1}, S_{n-2}}\left(a_{(n-1 n)}\right)$;

(4) $a_{(12)} N_{S_{2}, S_{1}}\left(a_{(23)}\right) N_{S_{3}, S_{2}}\left(a_{(34)}\right) \cdots N_{S_{n-1}, S_{n-2}}\left(a_{(n-1 n)}\right)$;

(5) $a_{(12)}\left(a_{(23)}+u^{-1} a_{(13)}\right)\left(a_{(34)}+u^{-1} a_{(24)}+u^{-2} a_{(14)}\right) \cdots\left(a_{(n-1 n)}+u^{-1} a_{(n-2 n)}+\right.$ $\left.\cdots+u^{-(n-2)} a_{(1 n)}\right)$.

Proof. Using Lemma (2.12) and the fact that $a_{(n-1 n)} \in Z_{A\left(S_{n}\right)}\left(A\left(S_{n-2}\right)\right)$, (2) can be derived from (1). Lemma (3.2) implies that $N_{S_{n-2}, S_{1}}\left(a_{(12)(23) \cdots(n-2 n-1)}\right) \in$ $Z\left(A\left(S_{n-1}\right)\right)$ so that (3) equals (2). Number (4) follows from (3) by induction, and (5) is just an expansion of (4).

Remark. Adopting the convention that $S_{0}=S_{1}=$ identity subgroup, representation (4) in Lemma (3.8) may be written as

$$
\prod_{i=1}^{n-1} N_{S_{i}, S_{i-1}}\left(a_{(i i+1)}\right) \text {. }
$$

By Proposition (2.13), $N_{S_{i}, S_{i-1}}\left(a_{(i i+1)}\right) \in Z_{A\left(S_{i+1}\right)}\left(A\left(S_{i}\right)\right)$. This implies that each factor in (3.9) commutes with every other factor in (3.9). Thus,

$$
\eta=N_{S_{n-1}, S_{n-2}}\left(a_{(n-1 n)}\right) N_{S_{n-2}, S_{n-3}}\left(a_{(n-2 n-1)}\right) \cdots N_{S_{2}, S_{1}}\left(a_{(23)}\right) a_{(12)} .
$$


Then an easy induction argument can be used to deduce that

$$
\begin{aligned}
& N_{S_{n-1}, S_{n-2}}\left(a_{(n-1 n)}\right) N_{S_{n-2}, S_{n-3}}\left(a_{(n-2 n-1)}\right) \cdots N_{S_{2}, S_{1}}\left(a_{(23)}\right) a_{(12)} \\
& \quad=N_{S_{n-1}, S_{1}}\left(a_{(n-1 n)(n-2 n-1) \cdots(23)(12)}\right) .
\end{aligned}
$$

Hence, $\eta=N_{S_{n-1}, S_{1}}\left(a_{(n-1 n)(n-2 n-1) \cdots(23)(12)}\right)$. This representation of $\eta$ will be used to prove a more general statement about $\eta$ in Lemma (3.16).

The following lemma, although trivial, contains observations which are helpful in giving clarity to later results.

Lemma (3.10). Let $\alpha \in S_{n}$ be an $n$-cycle. Then

(1) each reduced expression for a must involve all of the fundamental reflections of $S_{n}$,

(2) $l(\alpha) \geq n-1$,

(3) if $l(\alpha)=n-1$, each reduced expression for $\alpha$ involves each fundamental reflection of $S_{n}$ exactly once,

(4) if $l(\alpha)=n-1$, there is only one reduced expression for $\alpha$ up to rearrangement of the commuting fundamental reflections in $\alpha$.

Proof. If a reduced expression for $\alpha$ is missing a fundamental reflection of $S_{n}$, then $\alpha$, an $n$-cycle, would be living inside a parabolic subgroup of $S_{n}$. This is impossible since proper parabolic subgroups of $S_{n}$ are direct products of symmetric groups $S_{i}$ where $i<n$. This proves (1). Parts (2) and (3) follow easily from (1), and the fact that $S_{n}=\langle(12),(23), \ldots,(n-1 n)\rangle$. Part (4) is an immediate consequence of (3).

Lemma (3.11). Let $\alpha \in s_{n}$ with $l(\alpha)=n-1$. Suppose that a reduced expression for $\alpha$ involves every fundamental reflection of $S_{n}$ exactly once. Then $\alpha$ is an n-cycle.

Proof. Assume without loss of generality that the fundamental reflection $(n-1 n)$ appears to the right of $(n-2 n-1)$ in the reduced expression for $\alpha$. Since $(n-1 n)$ commutes with every other fundamental reflection of $S_{n}$, we may write

$$
\alpha=\alpha^{\prime}(n-1 n)
$$

with $\alpha^{\prime} \in S_{n-1}=\langle(12),(23), \ldots,(n-2 n-1)\rangle, \alpha^{\prime}$ reduced, $l\left(\alpha^{\prime}\right)=n-2$ and $\alpha^{\prime}$ involving every fundamental reflection of $S_{n-1}$ exactly once. By induction, $\alpha^{\prime}$ is an $(n-1)$-cycle. Write

$$
\alpha^{\prime}=\left(\pi_{1} \cdots n-1 \cdots \pi_{n-1}\right) .
$$

Then

$$
\begin{aligned}
\alpha & =\left(\pi_{1} \cdots n-1 \cdots \pi_{n-1}\right)(n-1 n) \\
& =\left(\pi_{1} \cdots n-1 n \cdots \pi_{n-1}\right)
\end{aligned}
$$

is an $n$-cycle.

Remark. Although there are redundancies in the hypotheses of Lemma (3.11), we have stated it this way for clarity. 
Definition (3.12). A partition of $n$ is a finite sequence of positive integers $\alpha=$ $\left(k_{1}, k_{2}, \ldots, k_{t}\right)$ such that

(1) $k_{i} \geq k_{i+1}$ for all $i$

(2) $\sum_{i} k_{i}=n$.

The fact that $\alpha$ is a partition of $n$ is abbreviated $\alpha \vdash n$.

If $\pi \in S_{n}$, then the ordered lengths of the cyclic factors of $\pi$ in cycle notation form a uniquely determined partition of $n$. Since cycle structure in $S_{n}$ uniquely determines conjugacy class, we have a one-to-one correspondence, in a natural way, between partitions of $n$ and conjugacy classes of $S_{n}$.

Lemma (3.13). Every conjugacy class in $S_{n}$, with the exception of the class of $n$-cycles, contains an element of length less than $n-1$.

Proof. Let $\alpha \vdash n$ with $\alpha=\left(k_{1}, k_{2}, \ldots, k_{t}\right), t>1$, so that $\alpha$ corresponds to a conjugacy class $C_{\alpha}$ of $S_{n}$ other than the class of $n$-cycles. Consider the element

$$
\begin{aligned}
\pi= & (12)(23) \cdots\left(k_{1}-1 k_{1}\right)\left(k_{1}+1 k_{1}+2\right)\left(k_{1}+2 k_{1}+3\right) \\
& \cdots\left(k_{1}+k_{2}-1 k_{1}+k_{2}\right) \\
& \cdots\left(k_{1}+k_{2}+\cdots+k_{t-1}+1 k_{1}+k_{2}+\cdots+k_{t-1}+2\right) \\
& \cdots\left(k_{1}+k_{2}+\cdots+k_{t}-1 k_{1}+k_{2}+\cdots+k_{t}\right) \\
= & \left(123 \cdots k_{1}\right)\left(k_{1}+1 k_{1}+2 \cdots k_{1}+k_{2}\right) \\
& \cdots\left(k_{1}+k_{2}+\cdots k_{t-1}+1 \cdots k_{1}+k_{2}+\cdots+k_{t}\right),
\end{aligned}
$$

where the convention is that $(r r)$ is the identity. Clearly $\pi \in C_{\alpha}$ and $l(\pi)=$ $\sum_{i=1}^{t}\left(k_{i}-1\right)=\sum_{i=1}^{t} k_{i}-t=n-t<n-1$. The lemma follows from Lemma (3.10), part (2).

Notes. (1) When we say an element $a_{\gamma}$ in $A\left(S_{n}\right)$ contains or involves a particular fundamental reflection, we mean that any reduced expression for $\gamma$ contains or involves that particular fundamental reflection in $S_{n}$.

(2) Given $a_{\gamma} \in A\left(S_{n}\right)$, the length of $a_{\gamma}$, denoted $l\left(a_{\gamma}\right)$, will mean the length of $\gamma, l(\gamma)$.

(3) Let $\alpha \in A\left(S_{n}\right)$. Then $\alpha$ may be written as

$$
\alpha=\sum_{u \in S_{n}} f_{w}(u) a_{w} \quad \text { with } f_{u}(u) \in \mathbf{Q}\left[u, u^{-1}\right] .
$$

By a term of $\alpha$ we mean $f_{w}(u) a_{w^{\prime}}$ for some $w \in S_{n}$, with $f_{u^{\prime}}(u) \neq 0$.

Lemma (3.14). Every term of $\eta$ contains each of the fundamental reflections (12), (23), .., $(n-1 n)$.

Proof. Appealing to Lemma (3.8), part (3), and expanding we have

$$
\begin{aligned}
\eta & =N_{S_{n-2}, S_{1}}\left(a_{(12)(23) \cdots(n-2 n-1)}\right) N_{S_{n-1}, S_{n-2}}\left(a_{(n-1 n)}\right) \\
& =N_{S_{n-2}, S_{1}}\left(a_{(12)(23) \cdots(n-2 n-1)}\right) \sum_{t=1}^{n-1} u^{-(l((n-t n))-1) / 2} a_{(n-t n)} .
\end{aligned}
$$


By induction, every term of $N_{S_{n-2}, S_{1}}\left(a_{(12)(23) \cdots(n-2 n-1)}\right)$ contains each of the fundamental reflections $(12),(23), \ldots,(n-2 n-1)$. Let $a_{\gamma}$ be an arbitrary term of $N_{S_{n-2}, S_{1}}\left(a_{(12)(23) \cdots(n-2 n-1)}\right)$, disregarding the coefficient. Let $\tau=$ $(n-1 n)(n-2 n-1) \cdots(n-t n-t+1)$ for a fixed $t$ with $1 \leq t \leq n-1$. Then

$$
a_{\gamma} \cdot a_{(n-t n)}=a_{\gamma} a_{(n-t n-t+1)(n-t+1 n-t+2) \cdots(n-2 n-1)} a_{\tau} .
$$

Each of the terms $f_{\lambda}(u) a_{\lambda}, f_{\lambda}(u) \neq 0$, of the product

$$
a_{\gamma} a_{(n-t n-t+1)(n-t+1 n-t+2) \cdots(n-2 n-1)}
$$

is such that $\lambda \in S_{n-1}$ with $\lambda$ involving all of the fundamental reflections of $S_{n-1}$ with the possible exception of $(n-t n-t+1),(n-t+1 n-t+2), \ldots$, $(n-2 n-1)$. But $\tau$ contains these possible deficiencies in addition to $(n-1 n)$, and since $\tau$ is a distinguished right coset representative for $S_{n-1}$ in $S_{n}$, we have by Proposition (1.1.2) that $a_{\lambda} a_{\tau}=a_{\lambda \tau}$ so that every term in $a_{\gamma} a_{(n-t n)}$ contains each of the fundamental reflections (12), (23), ., $(n-1 n)$. The lemma follows.

Corollary (3.15). Let $f_{\gamma}(u) a_{\gamma}$ be an arbitrary term in $\eta$ with $f_{\gamma}(u) \neq 0$. Then $l(\gamma) \geq n-1$.

Proof. Since there are $n-1$ fundamental reflections of $S_{n}$, the corollary is an immediate consequence of Lemma (3.14).

Lemma (3.16). Let $\tau \in S_{n}$ be an $n$-cycle with $l(\tau)=n-1$. Then

$$
N_{S_{n-1}, S_{1}}\left(a_{\tau}\right)=\eta \text {. }
$$

Proof. By Lemma (3.10), part (3), a reduced expression for $\tau$ involves every fundamental reflection of $S_{n}$ exactly once. Assume first that $(n-1 n)$ occurs to the right of $(n-2 n-1)$ in a reduced expression for $\tau$. Since $(n-1 n)$ commutes with every other fundamental reflection of $S_{n}$, we may write

$$
\tau=\tau^{\prime}(n-1 n),
$$

where $\tau^{\prime}$ is reduced, $l\left(\tau^{\prime}\right)=n-2$ and $\tau^{\prime}$ involves each of the fundamental reflections $(12),(23), \ldots,(n-2 n-1)$ exactly once. Thus Lemma (3.11) implies that $\tau^{\prime}$ is an $(n-1)$-cycle. Therefore

$$
\begin{aligned}
N_{S_{n-1}, S_{1}}\left(a_{\tau}\right) & =N_{S_{n-1}, S_{1}}\left(a_{\tau^{\prime}} a_{(n-1 n)}\right) \\
& =N_{S_{n-1}, S_{n-2}}\left(N_{S_{n-2}, S_{1}}\left(a_{\tau^{\prime}}\right) a_{(n-1 n)}\right) .
\end{aligned}
$$

Since this lemma is trivial for $n=2$, by induction we have $N_{S_{n-2}, S_{1}}\left(a_{\tau^{\prime}}\right)=$ $N_{S_{n-2}, S_{1}}\left(a_{(12)(23) \cdots(n-2 n-1)}\right)$. Hence

$$
\begin{aligned}
N_{S_{n-1}, S_{1}}\left(a_{\tau}\right) & =N_{S_{n-1}, S_{n-2}}\left(N_{S_{n-2}, S_{1}}\left(a_{(12)(23) \cdots(n-2 n-1)}\right) a_{(n-1 n)}\right) \\
& =\eta \quad \text { by Lemma }(3.8) .
\end{aligned}
$$


Now assume that $(n-1 n)$ occurs to the left of $(n-2 n-1)$ in a reduced expression for $\tau$. Following the same line of reasoning as above, we may write

$$
\tau=(n-1 n) \tau^{\prime}
$$

where $\tau^{\prime}$ is reduced, $l\left(t^{\prime}\right)=n-2$ and $\tau^{\prime}$ involves each of the fundamental reflections $(12),(23), \ldots,(n-2 n-1)$ exactly once. As before, Lemma (3.11) implies $\tau^{\prime}$ is an $(n-1)$-cycle and we have

$$
\begin{aligned}
N_{S_{n-1}, S_{1}}\left(a_{\tau}\right) & =N_{S_{n-1}, S_{1}}\left(a_{(n-1 n)} a_{\tau^{\prime}}\right) \\
& =N_{S_{n-1}, S_{n-2}}\left(a_{(n-1 n)} N_{S_{n-2}, S_{1}}\left(a_{\tau^{\prime}}\right)\right) .
\end{aligned}
$$

By induction we have

$$
N_{S_{n-2}, S_{1}}\left(a_{\tau^{\prime}}\right)=N_{S_{n-2}, S_{1}}\left(a_{(n-2 n-1)(n-3 n-2) \cdots(23)(12)}\right) .
$$

Thus

$$
\begin{aligned}
N_{S_{n-1}, S_{1}}\left(a_{\tau}\right) & =N_{S_{n-1}, S_{n-2}}\left(a_{(n-1 n)} N_{S_{n-2}, S_{1}}\left(a_{(n-2 n-1) \cdots(12)}\right)\right) \\
& =N_{S_{n-1}, S_{n-2}}\left(N_{S_{n-2}, S_{1}}\left(a_{(n-1 n)(n-2 n-1) \cdots(12)}\right)\right. \\
& =N_{S_{n-1}, S_{1}}\left(a_{(n-1 n)(n-2 n-1) \cdots(23)(12)}\right) \\
& =\eta
\end{aligned}
$$

by the remark following Lemma (3.8).

Lemma (3.17). Let $f_{\gamma}(u) a_{\gamma}$ be a term of $\eta$ with $f_{\gamma}(u) \neq 0$. Suppose $l(\gamma)=$ $n-1$. Then $\gamma$ is an $n$-cycle.

Proof. In taking the norm of $a_{(12)(23) \cdots(n-1 n)}$ to get $\eta$, the lengths for the right multiplications add by Proposition (1.1.2). Hence, the only way to get back down to an element $a_{\gamma}$, with $l(\gamma)=n-1$, is to have every fundamental reflection coming from the corresponding element being multiplied on the left reduce the length. That is, $\gamma$ must be of the form $r^{-1}(12)(23) \cdots(n-1 n) r$ which is of course an $n$-cycle.

Lemma (3.18). Let $\pi \in S_{n}$ be an $n$-cycle with $l(\pi)=n-1$. Then $a_{\pi}$ occurs with coefficient one in the complete expansion of $\eta$.

Proof. Lemma (3.10), part (3), implies that $(n-1 n)$ occurs in any reduced expression for $\pi$. Assume that $(n-1 n)$ occurs to the right of $(n-2 n-1)$ in a reduced expression for $\pi$. So we may write

$$
\pi=\pi^{\prime}(n-1 n)
$$

where $\pi^{\prime}$ is reduced, $l\left(\pi^{\prime}\right)=n-2$ and $\pi^{\prime}$ involves each of the fundamental reflections $(12),(23), \ldots,(n-2 n-1)$ exactly once. Hence, by Lemma (3.11), 
$\pi^{\prime}$ is an $(n-1)$-cycle. Then using Lemmas (3.8) and (3.16), we get

$$
\begin{aligned}
\eta & =N_{S_{n-1}, S_{n-2}}\left(N_{S_{n-2}, S_{1}}\left(a_{\pi^{\prime}}\right) a_{(n-1 n)}\right) \\
& =N_{S_{n-1}, S_{n-2}}\left(N_{S_{n-2}, S_{1}}\left(a_{(12)(23) \cdots(n-2 n-1)}\right) a_{(n-1 n)}\right) \\
& =N_{S_{n-2}, S_{1}}\left(a_{(12)(23) \cdots(n-2 n-1)}\right) N_{S_{n-1}, S_{n-2}}\left(a_{(n-1 n)}\right) \\
& =N_{S_{n-2}, S_{1}}\left(a_{(12)(23) \cdots(n-2 n-1)}\right) \sum_{t=1}^{n-1} u^{-(l((t n))-1) / 2} a_{(t n)} .
\end{aligned}
$$

From this we see that to get the term $a_{\pi}$ there must be some $\gamma \in S_{n-1}$ such that

$$
\gamma(k n)=\pi=\pi^{\prime}(n-1 n) \text { for some } 1 \leq k \leq n-1 .
$$

This implies that

$$
(k n)(n-1 n)=\gamma^{-1} \pi^{\prime} \in S_{n-1}
$$

which is a contradiction unless $k=n-1$. Thus $\gamma=\pi^{\prime}$. This lemma holds obviously for $n=2$ and so we assume by induction that $a_{\pi^{\prime}}$ occurs with coefficient one in $N_{S_{n-2}, S_{1}}\left(a_{(12)(23) \cdots(n-2 n-1)}\right)$. This forces the coefficient of $a_{\pi}$ to be one in $\eta$. The case when $(n-1 n)$ occurs to the left of $(n-2 n-1)$ in a reduced expression for $\pi$ is handled in a similar manner.

Notation. Let $\alpha \in A\left(S_{n}\right)$. We will write $\left.\alpha\right|_{u=1}$ to mean the image of $\alpha$ in the specialization of $A\left(S_{n}\right)$ at $u=1$. We say that $\alpha$ is specialized at $u=1$.

Lemma (3.19). Utilizing the fact that $\left.A\left(S_{n}\right)\right|_{u=1} \simeq \mathbf{Q} S_{n}$, we have that $\left.\eta\right|_{u=1}$ equals the sum of the $n$-cycles in $\mathbf{Q} S_{n}$, making the natural identification of $a_{w}$ in $A\left(S_{n}\right)$ with $w$ in $S_{n}$.

Proof. Since $\left.\eta\right|_{u=1} \in Z\left(\mathbf{Q} S_{n}\right)$, it may be written as a $\mathbf{Q}$-linear combination of conjugacy class sums for $S_{n}$. Using Lemma (3.13) and Corollary (3.15) we conclude that $\left.\eta\right|_{u=1}$ must be a $\mathbf{Q}$-multiple of the single class sum of $n$-cycles. By Lemma (3.18), this multiple is one and the lemma is proved.

Remark. Lemma (3.19) follows easily from the observation that

$$
\begin{aligned}
\left.\eta\right|_{u=1} & =\left.N_{S_{n-1}, S_{1}}\left(a_{(12)(23) \cdots(n-1 n)}\right)\right|_{u=1} \\
& =N_{S_{n-1}, S_{1}}((12)(23) \cdots(n-1 n)),
\end{aligned}
$$

which is the class sum of $n$-cycles in $S_{n}$. The proof given above is more consistent with the general methods of proof given for other results in this section.

Definition (3.20). For each partition $\alpha=\left(k_{1}, k_{2}, \ldots, k_{t}\right)$ of $n$ (recall $k_{1} \geq$ $k_{2} \geq \cdots \geq k_{t}$ ), we say a parabolic subgroup $P_{\alpha, s}$ of $S_{n}$ corresponds to $\alpha$ if

$$
P_{c, s}=S_{k_{1}} \times S_{k_{2}} \times \cdots \times S_{k_{t}},
$$

where $s$ is a numeric subscript used to distinguish among the different parabolic subgroups of $S_{n}$ having the shape of $\alpha$. For example, for $n=5, P_{\alpha, 1}=$ 
$\langle(12)\rangle \times\langle(34)\rangle \times S_{1}$ and $P_{\alpha, 2}=\langle(23)\rangle \times\langle(45)\rangle \times S_{1}$ both have shape $\alpha=(2,2,1)$. In some sense there is a natural representative parabolic subgroup to choose from among all parabolic subgroups having shape $\alpha$, namely,

$$
S_{k_{1}} \times S_{k_{2}} \times \cdots \times S_{k_{t}}
$$

with

$$
\begin{aligned}
& S_{k_{1}}=\left\langle(12),(23), \ldots,\left(k_{1}-1 k_{1}\right)\right\rangle, \\
& S_{k_{2}}=\left\langle\left(k_{1}+1 k_{1}+2\right),\left(k_{1}+2 k_{1}+3\right), \ldots,\left(k_{1}+k_{2}-1 k_{1}+k_{2}\right)\right\rangle
\end{aligned}
$$

and so on. We will let $P_{x, 1}$ be this particular left-justified choice and denote it simply as $P_{\alpha}$, deleting the subscript $s$.

Definition (3.21). Let $P_{\alpha, s}$ be a parabolic subgroup of $S_{n}$ corresponding to a partition $\alpha=\left(k_{1}, k_{2}, \ldots, k_{t}\right)$ of $n$ as in Definition (3.20). Define

$$
\begin{aligned}
\eta_{\alpha, S} & =\prod_{i=1}^{t} N_{S_{k_{i}-1}, S_{1}}\left(a_{w_{i}}\right), \\
l_{c k} & =\sum_{i=1}^{t}\left(k_{i}-1\right),
\end{aligned}
$$

where $s$ is as it was in Definition (3.20), and where $w_{i}$ is a $k_{i}$-cycle in $S_{k_{i}}$ of length $k_{i}-1$ and $N_{S_{k_{i}-1}, S_{1}}\left(a_{w_{i}^{\prime}}\right)=a_{1}$ if $k_{i}=1$. Also, $\eta_{\alpha}=\eta_{\alpha, 1}$.

Remarks. (1) For $\alpha=(n)$, there is of course only one $P_{\alpha, s}$, namely $P_{\alpha}=S_{n}$. So then $\eta_{\alpha, s}=\eta_{t}=\eta$.

(2) If a fundamental reflection appears in one of the norm factors of $\eta_{\alpha, s}$ it will not appear in any other factor. This implies that these factors commute with each other and the lengths add in the multiplications.

Lemma (3.22). Let $\alpha, \beta \in A\left(S_{n}\right)$. Then

(1) $\left.(\alpha+\beta)\right|_{u=1}=\left.\alpha\right|_{u=1}+\left.\beta\right|_{u=1}$,

(2) $\left.(\alpha \beta)\right|_{u=1}=\left.\left.\alpha\right|_{u=1} \cdot \beta\right|_{u=1}$,

Proof. (1) This is clear.

(2) In view of (1), we only need to consider the trivial case $\alpha=f(u) a_{u v}$, $\beta=g(u) a_{u u^{\prime}}$, where $w, w^{\prime} \in S_{n}$. Then

$$
\begin{aligned}
\left.\alpha \beta\right|_{u=1} & =\left.f(u) g(u) a_{u^{\prime}} a_{u^{\prime}}\right|_{u=1} \\
& =\left.f(u) g(u)\left(u^{z} a_{u^{\prime} u^{\prime}}+(u-1)(\text { possible other terms })\right)\right|_{u=1} \\
& =f(1) g(1) a_{w^{\prime} u^{\prime}}=f(1) a_{w} g(1) a_{u^{\prime}}=\left.\left.\alpha\right|_{u=1} \cdot \beta\right|_{u=1} . \quad \square
\end{aligned}
$$

Lemma (3.23). Let $\alpha, P_{\alpha, s}, \eta_{\alpha, s}$ and $l_{c,}$ be as in Definition (3.21). Then

(1) $\eta_{c, s}$ involves only terms of length $l_{c}$ and longer,

(2) the coefficient on all terms corresponding to elements in the class of $\alpha$ of length $l_{\text {c }}$ is one,

(3) $\eta_{r, s} \in Z\left(A\left(P_{r, s}\right)\right)$, 
(4) $\left.\eta_{\alpha, s}\right|_{u=1}=\prod_{i=1}^{t}$ (sum of the $k_{i}$-cycles in $\left.S_{k_{i}}\right)$,

(5) Let $f_{\gamma}(u) a_{\gamma}, f_{\gamma}(u) \neq 0$, be a term of $\eta_{\alpha, s}$ with $l(\gamma)=l_{\alpha}$. Then $\gamma \in$ $C_{\alpha} \cap P_{\alpha, s}$ (consequently, $f_{\gamma}(u)=1$ by part (2) of this lemma).

Proof. (1) This follows from Corollary (3.15), Lemma (3.16), and Remark (2) following Definition (3.21).

(2) This follows from Lemma (3.16), Lemma (3.18), and Remark (2) following Definition (3.21).

(3) This follows from Lemma (3.2) and Remark (2) following Definition (3.21).

(4) This follows from Lemma (3.19) and Lemma (3.22).

(5) This follows from Lemma (3.17) and Remark (2) following Definition (3.21).

Remarks. (1) We may restate part (4) of Lemma (3.23) as follows: Let $C_{\alpha}$ be the conjugacy class in $S_{n}$ corresponding to $\alpha$. Then

$$
\left.\eta_{\alpha, s}\right|_{u=1}=\text { the sum of the elements in } C_{\alpha} \cap P_{\alpha, s} .
$$

(2) If we write $\eta_{\alpha, s}=\sum_{w \in P_{u}, s} f_{w}(u) a_{w}$ with $f_{w}(u) \in \mathbf{Q}\left[u, u^{-1}\right]$, part (4) implies $f_{u}(1)=0$ or 1 .

Definition (3.24). Let $\alpha, P_{\alpha, s}$, and $\eta_{\alpha, s}$ be as in Definition (3.21). Define

$$
b_{\alpha, s}=N_{S_{n}, P_{\alpha, s}}\left(\eta_{\alpha, s}\right) \text {. }
$$

Also,

$$
b_{\alpha}=N_{S_{n}, P_{n}}\left(\eta_{\alpha}\right) .
$$

Lemma (3.25). The element $b_{\alpha, s}$ contains only terms of length $l_{\alpha}$ and longer. Proof. In taking the norm of $\eta_{\alpha, s}$, lengths for right multiplications add by Proposition (1.1.2). The left multiplictions can only reduce the length by, at most, the same length as it had been increased by the right multiplications. Thus the result follows from Corollary (3.15).

Lemma (3.26). The element $b_{\alpha, s}$ is in $Z(A)$.

Proof. This is immediate from Lemma (3.23), part (3) and Proposition (2.13).

Lemma (3.27). Let $f_{\gamma}(u) a_{\gamma}$ be a term of $b_{\alpha, s}$ with $f_{\gamma}(u) \neq 0$. Suppose $l(\gamma)=$ $l_{\alpha}$. Then $\gamma \in C_{\alpha}$.

Proof. In view of Lemma (3.23), part (5), we can use the same argument on a term $a_{\gamma}$ of $\eta_{\alpha, s}$ with $l(\gamma)=l_{\alpha}$ in taking the norm of $\eta_{\alpha, s}$ to get $b_{\alpha, s}$, as was used in Lemma (3.17), to get this result.

Corollary (3.28). Let $f_{\gamma}(u) a_{\gamma}$ be a term of $b_{\alpha, s}$ with $l(\gamma)=l_{\alpha}$. Then $f_{\gamma}(u)$ is a positive integer.

Proof. From Lemma (3.27) and the technique in its proof, $\gamma \in C_{\alpha}$ and $a_{\gamma}$ must be realized as $a_{r-1 \tau r}$ where $r$ is a distinguished right coset representative 
for $P_{\alpha, s}$ in $S_{n}, \tau \in C_{\alpha} \cap P_{\alpha, s}$ with $l(\tau)=l_{\alpha}$ and $a_{\tau}$ is a term of $\eta_{\alpha, s}$ having coefficient one. Thus, in taking the norm we get the product

$$
u^{-l(r)} a_{r^{-1}} a_{\tau} a_{r}=a_{\gamma}+\text { other terms of longer length }
$$

and the corollary follows.

Proposition (3.29). Let $C_{\alpha}$ be the conjugacy class of $S_{n}$ corresponding to $\alpha$ and let $\underline{C}_{\alpha}$ denote the sum of the elements in $C_{\alpha}$. Then

$$
\left.b_{\alpha, s}\right|_{u=1}=\left[N_{S_{n}}\left(P_{\alpha, s}\right): P_{\alpha, s}\right] \underline{C}_{\alpha} .
$$

Proof. Let $f_{\gamma}(u) a_{y}$, be a term of $\eta_{\alpha, s}$ with $f_{\gamma}(1) \neq 0$ so that $\gamma \in C_{\alpha} \cap P_{\alpha, s}$ by Remark (1) following Lemma (3.23). Let $T$ be the set of distinguished right coset representatives for $P_{\alpha, s}$ in $S_{n}$. Then for $r \in T$, we have

$$
a_{r^{-1}} f_{\gamma^{\prime}}(u) a_{\gamma} a_{r}=f_{\gamma}(u) a_{r^{-1} \gamma r}+f_{\gamma}(u)(u-1)(\text { other terms) }
$$

so that only terms like $a_{r^{-1} \gamma r}$ will survive in the specialization of $b_{\alpha, s}$; that is, only terms corresponding to conjugates of $\gamma$. From Lemma (3.26) we conclude that $\left.b_{\alpha, s}\right|_{u=1}$ is some multiple of $\underline{C}_{\alpha}$. To determine the exact multiple, we will determine the coefficient on $a_{\gamma}$ in $\left.b_{\alpha, s}\right|_{u=1}$. If $r \in N_{S_{n}}\left(P_{\alpha, s}\right) \cap T$, then by Lemma (3.22), part (2), and Lemma (3.23), part (4), we have that $\left.a_{r^{-1}} \eta_{\alpha, s} a_{r}\right|_{u=1}$ contains exactly one $a_{\gamma}$. On the other hand, if for $\lambda \in C_{\alpha} \cap P_{\alpha, s}$ and $r \in T$ we have $r^{-1} \lambda r=\gamma$, then $w^{-1} r^{-1} \lambda r w=w^{-1} \gamma \omega=\lambda$ for some $w \in P_{\alpha, s}$. Thus $r w \in C_{S_{n}}(\lambda)$. Rewrite $P_{\alpha, s}$ as $S_{m_{1}}^{v_{1}} \times S_{m_{2}}^{v_{2}} \times \cdots \times S_{m_{z}}^{v_{z}}$ where $S_{m_{i}} \not S_{m_{j}}$ for $i \neq j$. Then write $\lambda$ as $\prod_{j=1}^{z} \prod_{i=1}^{v_{j}} \lambda_{i, j}$ where $\lambda_{i, j}$ is an $m_{j}$-cycle of length $m_{j}-1$ inside one of the $S_{m_{j}}$ factors of $P_{\alpha, s}$. Thus

$$
\begin{aligned}
C_{S_{n}}(\lambda) & =S_{t} \times \prod_{j=1}^{z} \prod_{i=1}^{v_{j}}\left\langle\lambda_{i, j}\right\rangle \times S_{v_{1}} \times S_{v_{2}} \times \cdots \times S_{v_{z}} \\
& \leq S_{t} \times P_{\alpha, s} \times S_{v_{1}} \times S_{v_{2}} \times \cdots \times S_{v_{z}}^{z} \\
& =N_{S_{n}}\left(P_{\alpha, S}\right), \quad \text { where } t=n-\sum_{i=1}^{z} v_{i} m_{i} .
\end{aligned}
$$

Therefore, $r w \in N_{S_{n}}\left(P_{\alpha, s}\right)$ and since obviously $w \in N_{S_{n}}\left(P_{\alpha, s}\right)$, it follows that $r \in N_{S_{n}}\left(P_{\alpha, s}\right)$. Hence, the coefficient on $a_{\gamma}$ in $\left.b_{\alpha, s}\right|_{u=1}$ is $\left|N_{S_{n}}\left(P_{\alpha, s}\right) \cap T\right|=$ $\left[N_{S_{n}}\left(P_{c, s}\right): P_{\alpha, s}\right]$ and the proposition is proved.

Corollary (3.30). The positive integer coefficient that occurs in Corollary (3.28) is $\left[N_{S_{n}}\left(P_{\alpha, s}\right): P_{\alpha, s}\right]$.

Proof. This is immediate from Corollary (3.28) and Proposition (3.29).

Remark. Since $\left[N_{S_{n}}\left(P_{\alpha, s}\right): P_{\alpha, s}\right]=\left[N_{S_{n}}\left(P_{\alpha}\right): P_{\alpha}\right]$, the positive integer coefficient in Corollary (3.28) is independent of $s$. 
Definition (3.31). Define $B$ to be the set $\left\{b_{\alpha} \mid \alpha \vdash n\right\}$, and $\left.B\right|_{u=1}$ to be the set $\left\{\left.b_{\alpha}\right|_{u=1} \mid \alpha \vdash n\right\}$.

Corollary (3.32). The set $\left.B\right|_{u=1}$ is a basis for $Z\left(\mathbf{Q} S_{n}\right)$ over $\mathbf{Q}$.

Proof. Immediate from Proposition (3.29).

Remark. Lemma (3.25) and Lemma (3.27) imply that the elements of $B$ can be put into a triangular form with respect to the lengths of the terms involved in the elements of $B$. This fact is crucial to the proof of the following main theorem.

Theorem (3.33). The set $B$ is a basis for $Z\left(A\left(S_{n}\right)\right)$ over $\mathbf{Q}\left[u, u^{-1}\right]$.

Proof. Suppose there is a relation $\sum_{\alpha \vdash n} f_{\alpha}(u) b_{\alpha}=0$ with $f_{\alpha}(u) \in \mathbf{Q}\left[u, u^{-1}\right]$ and not all $f_{\alpha}(u)$ equal to zero. If $f_{\alpha}(1)=0$ for all $f_{\alpha}(u) \neq 0$, then $u-1$ is a factor of each $f_{c}(u) \neq 0$ and we may cancel it. Thus we may assume that $f_{\alpha}(1) \neq 0$ for some $\alpha$. But then

$$
0=\left.\left(\sum_{\alpha \vdash n} f_{\alpha}(u) b_{\alpha}\right)\right|_{u=1}=\left.\sum_{\alpha \vdash n} f_{\alpha}(1) b_{\alpha}\right|_{u=1}
$$

contradicts Corollary (3.32). Hence, $B$ is a linearly independent set over $\mathbf{Q}\left[u, u^{-1}\right]$. Now, for each partition $\alpha=\left(k_{1}, k_{2}, \ldots, k_{t}\right)$ of $n$, pick an element $\beta_{\alpha, \tau}=a_{\tau} \in\left\{a_{w} \mid w \in S_{n}\right\}$ such that $\tau \in C_{\alpha}, l(\tau)=l_{\alpha}$ and $\tau$ is supported on $\left\{1,2, \ldots, \sum_{i=1}^{v} k_{i}\right\}$ where $k_{i}=1$ for $v<i \leq t$. In other words, $\tau$ has minimal length in its conjugacy class in $S_{n}$ and is left-justified. Let $S=\left\{\beta_{\alpha, \tau} \mid \alpha \vdash n\right\}$. Denote the span of $S$ in $A\left(S_{n}\right)$ over $\mathbf{Q}\left[u, u^{-1}\right]$ by $\langle S\rangle$. Let $\pi$ be the projection of $Z\left(A\left(S_{n}\right)\right)$ onto $\langle S\rangle$. Considering dimensions over $\mathbf{Q}(u)$, we deduce from the fact mentioned in the remark following Theorem (1.2.5) that the kernel of $\pi$ is trivial. Thus

$$
\langle S\rangle / \pi(\langle B\rangle) \simeq Z\left(A\left(S_{n}\right)\right) /\langle B\rangle .
$$

From Lemma (3.25) and Lemma (3.27) we have $\pi(B)=S$ so that $\pi(\langle B\rangle)=$ $\langle S\rangle$. Hence, $\langle B\rangle=Z\left(A\left(S_{n}\right)\right)$ and the theorem is proved.

Examples. (1) $n=3$.

$$
\eta=N_{S_{2}, S_{1}}\left(a_{(12)(23)}\right)=a_{(123)}+a_{(132)}+u^{-1}(u-1) a_{(13)} .
$$

Let $\beta=a_{(12)}+a_{(23)}+u^{-1} a_{(13)}$. Then

$$
B=\left\{N_{S_{3}, S_{1}}\left(a_{1}\right), N_{S_{3}, S_{2}}\left(a_{(12)}\right), N_{S_{3}, S_{3}}(\eta)=\eta\right\},
$$

where

$$
N_{S_{3}, S_{1}}\left(a_{1}\right)=6 a_{1}+3 u^{-1}(u-1) \beta+u^{-2}(u-1)^{2} \eta
$$

and

$$
N_{S_{3}, S_{2}}\left(a_{(12)}\right)=\beta+u^{-1}(u-1) \eta .
$$


(2) $n=4$.

$$
\begin{aligned}
\eta= & N_{S_{3}, S_{1}}\left(a_{(12)(23)(34)}\right) \\
= & a_{(1234)}+a_{(1432)}+a_{(1243)}+a_{(1342)} \\
& +u^{-2}\left(u^{2}-u+1\right)\left(a_{(1423)}+a_{(1324)}\right) \\
& +u^{-1}(u-1)\left(a_{(13)(24)}+a_{(124)}+a_{(142)}+a_{(134)}+a_{(143)}\right) \\
& +u^{-2}(u-1)^{2} a_{(14)}+u^{-3}(u-1)\left(u^{2}+1\right) a_{(14)(23)}
\end{aligned}
$$

Let

$$
\begin{aligned}
\beta= & a_{(12)(34)}+u^{-1} a_{(13)(24)}+u^{-2} a_{(14)(23)} \\
\gamma= & a_{(123)}+a_{(132)}+a_{(234)}+a_{(243)} \\
& +u^{-1}\left(a_{(124)}+a_{(142)}+a_{(134)}+a_{(143)}\right) \\
& +u^{-2}(u-1)\left(a_{(1324)}+a_{(1423)}\right)+2 u^{-2}(u-1) a_{(14)} \\
& +u^{-3}(u-1)^{2} a_{(14)(23)}+u^{-1}(u-1)\left(a_{(13)}+a_{(24)}\right)
\end{aligned}
$$

and

Then

$$
\tau=a_{(12)}+a_{(23)}+a_{(34)}+u^{-1}\left(a_{(13)}+a_{(24)}\right)+u^{-2} a_{(14)} .
$$

$$
B=\left\{N_{S_{4}, S_{1}}\left(a_{1}\right), N_{S_{4}, S_{2}}\left(a_{(12)}\right), N_{S_{4}, S_{2} \times S_{2}}\left(a_{(12)(34)}\right), N_{S_{4}, S_{3}}\left(N_{S_{2}, S_{1}}\left(a_{(12)(23)}\right)\right), \eta\right\},
$$

where

$$
\begin{gathered}
N_{S_{4}, S_{1}}\left(a_{1}\right)=24 a_{1}+12 u^{-1}(u-1) \tau+4 u^{-2}(u-1)^{2} \gamma \\
+6 u^{-2}(u-1)^{2} \beta+u^{-3}(u-1)^{3} \eta \\
N_{S_{4}, S_{2}}\left(a_{(12)}\right)=2 \tau+2 u^{-1}(u-1) \gamma+2 u^{-1}(u-1) \beta+u^{-2}(u-1)^{2} \eta \\
N_{S_{4}, S_{2} \times S_{2}}\left(a_{(12)(34)}\right)=2 \beta+u^{-1}(u-1) \eta \\
N_{S_{4}, S_{3}}\left(N_{S_{2}, S_{1}}\left(a_{(12)(23)}\right)\right)=N_{S_{4}, S_{3}}\left(a_{(123)}+a_{(132)}+u^{-1}(u-1) a_{(13)}\right) \\
=\gamma+u^{-1}(u-1) \eta .
\end{gathered}
$$

\section{REFERENCES}

1. Michael Aschbacher, Finite group theory, Cambridge Univ. Press, 1986.

2. N. Bourbaki, Groupes et algèbres de Lie, Chapters 4-6, Actualités Sci. Indust., No. 1337, Hermann, Paris, 1968.

3. R. W. Carter, Simple groups of Lie type, Wiley, New York and London, 1972.

4. C. W. Curtis, Representations of finite groups of Lie type, Bull. Amer. Math. Soc. (N.S.) 1 (1979), 721-757.

5. __ On Lusztig's isomorphism theorem for Hecke algebras, J. Algebra 92 (1985), 348-364.

6. __ Representation theory notes from a course given at the University of Virginia in 1975. 
7. C. W. Curtis, N. Iwahori, and R. Kilmoyer, Hecke algebras and characters of parabolic type of finite groups with $(B, N)$-pairs, Inst. Hautes Études Sci. Publ. Math. 40 (1972), 81-116.

8. C. W. Curtis and I. Reiner, Representation theory of finite groups and associative algebras, Interscience, 1962.

9. ___ Methods of representation theory, Vol. I, Wiley-Interscience, New York, 1981.

10. __ Methods of representation theory, Vol. II, Wiley-Interscience, New York, 1987.

11. C. W. Curtis and L. L. Scott, unpublished work, 1974.

12. W. Feit, The representation theory of finite groups, North-Holland Mathematical Library, vol. 25, North-Holland, Amsterdam, 1982.

13. P. Hoefsmit, Representations of Hecke algebras of finite groups with $(B, N)$-pairs of classical type, Ph.D. Dissertation, University of British Columbia, Vancouver, 1974.

14. P. Hoefsmit and L. L. Scott, unpublished manuscript, 1976.

15. David Kazhdan and George Lusztig, Representations of Coxeter groups and Hecke algebras, Invent. Math. 53 (1979), 165-184.

16. R. Kolmoyer, Some irreducible complex representations of a finite group with a BN-pair, Ph.D. Dissertation, M.I.T., 1969.

17. Brigette Kromar and Thomas Schmid-Leissler, Die irreduzißlen komponenten des Permutationscharakters $1_{B}^{G}$ einer endlichen $(B, N)$-Parr-Gruppe $G$, Diplomarbeit, Tübingen, 1976.

18. Peter Landrock, Finite group algebras and their modules, London Math. Soc. Lecture Note Series, vol. 84, Cambridge Univ. Press, 1983.

Department of Mathematics and Computer Science, Shippensburg University, Shippensburg, Pennsylvania 17257 